\title{
Disruption of de novo purine biosynthesis in Pseudomonas fluorescens Pf0-1 leads to reduced biofilm formation and a reduction in cell size of surface-attached but not planktonic cells
}

Shiro Yoshioka, Peter D Newell

Pseudomonas fluorescens Pf0-1 is one of the model organisms for biofilm research. Our previous transposon mutagenesis study suggested a requirement for the de novo purine nucleotide biosynthesis pathway for biofilm formation by this organism. This study was performed to verify that observation and investigate the basis for the defects in biofilm formation shown by purine biosynthesis mutants. Constructing deletion mutations in 8 genes in this pathway, we found that they all showed reductions in biofilm formation that could be partly or completely restored by nucleotide supplementation or genetic complementation. We demonstrated that, despite a reduction in biofilm formation, more viable mutant cells were recovered from the surface-attached population than from the planktonic phase under conditions of purine deprivation. Analyses using scanning electron microscopy revealed that the surface-attached mutant cells were $25 \sim 30 \%$ shorter in length than WT, which partly explains the reduced biomass in the mutant biofilms. The laser diffraction particle analyses confirmed this finding, and further indicated that the WT biofilm cells were smaller than their planktonic counterparts. The defects in biofilm formation and reductions in cell size shown by the mutants were fully recovered upon adenine or hypoxanthine supplementation, indicating that the purine shortages caused reductions in cell size. Our results are consistent with surface attachment serving as a survival strategy during nutrient deprivation, and indicate that changes in the cell size may be a natural response of $P$. fluorescens to growth on a surface. Finally, cell sizes in WT biofilms became slightly smaller in the presence of exogenous adenine than in its absence. Our findings suggest that purine nucleotides or related metabolites may influence the regulation of cell size in this bacterium. 
1

2

3 Mutations disrupting the de novo purine biosynthesis pathway in Pseudomonas fluorescens

4 Pf0-1 lead to reduced biofilm formation and a reduction in cell size of surface-attached but 5 not planktonic cells

6

7 Shiro Yoshioka ${ }^{1, *}$ and Peter D. Newell ${ }^{2}$

8

$9{ }^{1}$ Institute for Molecular Science, National Institutes of Natural Sciences, 5-1, Higashiyama, 10 Myodaiji, Okazaki, Aichi 444-8787 Japan

$11{ }^{2}$ Department of Biological Sciences, Oswego State University of New York, Oswego, New York 1213126 USA

13

$14 *$ Corresponding author

15 Shiro Yoshioka

\section{7}

18

FAX: +81-564-59-5576 


\section{Abstract}

23 Pseudomonas fluorescens $\mathrm{Pf0}-1$ is one of the model organisms for biofilm research. Our 24 previous transposon mutagenesis study suggested a requirement for the de novo purine 25 nucleotide biosynthesis pathway for biofilm formation by this organism. This study was 26 performed to verify that observation and investigate the basis for the defects in biofilm formation 27 shown by purine biosynthesis mutants. Constructing deletion mutations in 8 genes in this 28 pathway, we found that they all showed reductions in biofilm formation that could be partly or 29 completely restored by nucleotide supplementation or genetic complementation. We 30 demonstrated that, despite a reduction in biofilm formation, more viable mutant cells were 31 recovered from the surface-attached population than from the planktonic phase under conditions 32 of purine deprivation. Analyses using scanning electron microscopy revealed that the surface33 attached mutant cells were $25 \sim 30 \%$ shorter in length than WT, which partly explains the reduced 34 biomass in the mutant biofilms. The laser diffraction particle analyses confirmed this finding, 35 and further indicated that the WT biofilm cells were smaller than their planktonic counterparts. 36 The defects in biofilm formation and reductions in cell size shown by the mutants were fully 37 recovered upon adenine supplementation, indicating that purine shortages caused reductions in 38 cell size. Our results are consistent with surface attachment serving as a survival strategy during 39 nutrient deprivation, and indicate that changes in the cell size may be a natural response of $P$. 40 fluorescens to growth on a surface. Our findings suggest that purine nucleotides or related 41 metabolites may influence the regulation of cell size in this bacterium. 
44

45

46

47

48

49

50

51

52

53

54

55

56

57

58

59

60

61

62

63

64

65

66

67

68

69

\section{Introduction}

ATP and GTP are the purine nucleotide triphosphates that are essential to drive many cellular processes in all living organisms. ADP and GDP are utilized as DNA precursors after being converted to the deoxy forms by ribonucleotide reductase (Neuhard and Nygaard, 1987). AMP and GMP are the dephosphorylated forms of the above nucleotides and synthesized either in a de novo synthesis pathway or in a salvage pathway (Neuhard and Nygaard, 1987). In the de novo purine biosynthesis pathway, inosine monophosphate (IMP) is sequentially synthesized from 5-phosphoribosyl- $\alpha$-diphospahte (PRPP) in 11 enzymatic steps, where some reactions require ATP to proceed. AMP and GMP are synthesized separately from IMP as the common intermediate. Therefore, the biosynthesis of purine nucleotides has a high energy cost. For this reason, another biosynthesis pathway, salvage pathway, is designed to scavenge and recycle the purine bases arising from nucleic acid turnover; adenine, guanine, and hypoxanthine are converted into AMP, GMP and IMP, respectively, by phosphoribosyltransferases (Neuhard and Nygaard, 1987). The fact that most bacteria possess both de novo purine biosynthesis pathway and salvage pathway indicates vital role of this pathway in bacteria.

The importance of the de novo purine biosynthesis in bacterial growth has been repeatedly described in the literature. If one of the genes in de novo purine biosynthesis pathway is disrupted, the mutant becomes purine auxotroph. In other words, the mutant is not able to grow unless the exogenous purine bases such as adenine and hypoxanthine are supplied. Purine requiring mutants of some pathogenic bacteria have been found to be avirulent in murine models of infection, implying that the purine requiring mutants stop growing when exogenous purines are not available at the sites of infection, leading to attenuated infection (Bacon, Burrows \& Yates, 1951; Gerber, Hackett \& Franklin, 1952; Straley \& Harmon, 1984; Wang et al, 1996; Polissi et al., 1998; Pilatz et al., 2006; Samant et al., 2008; Jenkins et al., 2011). Furthermore, recent research has highlighted the role of the purine nucleotide biosynthesis on biofilm formation and symbiosis with nematode, insect or plant roots (Han et al., 2006; Ge et al., 2008; An and Grewal, 2011; Kim et al., 2014a). In these studies, significant reductions in biofilm formation and defects in symbiotic ability were observed for the purine auxotrophic mutants, emphasizing important roles of the purine biosynthesis pathway in biofilm formation and symbiosis (Han et al., 2006; An and Grewal, 2011; Ge et al., 2008; Kim et al., 2014a).

The purine nucleotide derivative c-di-GMP is a central player in the regulation of biofilm formation. Generally, increase in cellular level of c-di-GMP facilitates biofilm formation. This 
76

77

78

79

80

81

82

83

84

85

86

87

88

89

90

91

92

93

94

95

96

97

98

99

100

101

102

103

104

105

106

107

compound is synthesized from two molecules of GTP by diguanylate cyclases (DGCs) possessing GGDEF domain (Paul et al., 2004; Ryjenkov et al., 2005), and degraded by phosphodiesterases (PDEs) containing either EAL or HD-GYP domain (Christen et al., 2005; Schmidt, Ryjenkov \& Gomersky, 2005; Ryan et al., 2006). As these enzymes typically contain regulatory domains, the synthesis and degradation of c-di-GMP is influenced by environmental factors.

While DGCs and PDEs have the primary role in controlling the c-di-GMP level, previous work suggested that nucleotide pools impact c-di-GMP levels (Monds et al., 2010; Kim et al., 2014b). The disruption of the purT gene in the de novo purine biosynthesis pathway decreased cellular concentration of c-di-GMP, leading to defect in the biofilm formation by Burkholderia species (Kim et al., 2014b). Increase in c-di-GMP level was observed for the apaH mutant of Psudomonas fluorescens Pf0-1, which is caused by promotion of the de novo purine biosynthesis through increased level of di-adenosine tetraphosphate (Ap4A) (Monds et al., 2010). As for the purine auxotrophic mutants of $P$. fluorescens, biofilm formation could be impaired because the cellular concentrations of c-di-GMP may be decreased due to a reduced pool of GTP.

P. fluorescens Pf0-1 is a soil bacterium that promotes plant growth by forming biofilms on roots (Haas and Defago, 2005). This bacterium forms a biofilm using LapA, a large cell-surface adhesion protein with a molecular weight of $\sim 520 \mathrm{kDa}$ (Hinsa et al., 2003). The secretion and localization of LapA is regulated by LapD that binds c-di-GMP (Monds et al., 2007; Newell, Monds \& O'Toole, 2009). To identify the DGC genes involved in biofilm formation by this bacterium, we previously performed transposon mutagenesis and found that more than 50 genes are involved in the biofilm formation (Newell et al., 2011b). Among the mutants impaired in biofilm formation, the transposon insertions occurred in the purH, purL, purM, purF, and purK genes in de novo purine nucleotide biosynthesis pathway (Table S3 in Newell et al., 2011b). We therefore hypothesized that the de novo purine biosynthesis pathway is essential for the biofilm formation by this bacterium, as reported for other bacteria.

In this study, we sought to determine the basis for the biofilm formation defects observed in these mutants. To verify the requirement of the purine biosynthesis genes in biofilm formation, we constructed clean deletion mutants and performed functional complementation with exogenous genes and purine bases. Using electron scanning microscopy and a laser diffraction particle analyzer, we demonstrate that the surface-attached cells have smaller cell size compared to the planktonic cells and that the biofilm cells for the purine-depleted mutants became smaller 
108 than the WT cells. These data suggest purine auxotrophs of $P$. fluorescens are capable of

109 surface attachment, but produce less biofilm biomass due to the impact of purine deprivation on 110 growth and cell size.

111 


\section{Materials \& Methods}

113 Strains and media. Strain SMC4798 was used as the wild-type (WT) P. fluorescens Pf0-1,

114 which expresses fully functional three-hemagglutinin (HA)-tagged LapA (Monds et al., 2007;

115 Newell, Monds, \& O’Toole, 2009; Newell et al., 2011b; Boyd et al., 2014). Escherichia coli

116 S17-1 ( $\lambda$ pir) was used for cloning and conjugation. P. fluorescens and E. coli were routinely

117 cultured with LB medium in a test tube or on a solidified LB medium with $1.5 \%$ agar at $30^{\circ} \mathrm{C}$

118 and $37^{\circ} \mathrm{C}$, respectively. Saccharomyces cerevisiae strain InvSc1 (Life Technologies) was used to

119 construct plasmids for clean deletion and complementation, as previously described (Shanks et

120 al., 2006). Gentamycin (Gm) was used at $30 \mu \mathrm{g} / \mathrm{ml}$ for P. fluorescens and at $10 \mu \mathrm{g} / \mathrm{ml}$ for E. coli.

121 Chloramphenicol $(\mathrm{Cm})$ was used at $30 \mu \mathrm{g} / \mathrm{ml}$. For biofilm assay, K10T-1 medium that has been

122 used in this laboratory and consisted of $50 \mathrm{mM}$ Tris- $\mathrm{HCl}(\mathrm{pH} 7.4), 0.2 \%$ Bacto tryptone, $0.15 \%$

123 glycerol, 0.61 $\mathrm{mM} \mathrm{MgSO}_{4}$, and $1 \mathrm{mM} \mathrm{K}_{2} \mathrm{HPO}_{4}$, was used (Monds et al., 2006). Purine bases were

124 added to K10T-1 to a final concentration of $0.2 \mathrm{mM}$. Arabinose was used to induce expression of

125 the $\mathrm{P}_{\mathrm{BAD}}$ promoter from $\mathrm{pMQ} 72$ vector at a final concentration of $0.1 \%(\mathrm{wt} / \mathrm{vol})$.

126 Biofilm formation assay. Biofilm formation assays were performed using a polyvinyl

127 chloride 96-well round-bottom microtiter plate (Corning 2797). Aliquots (1.5 $\mu 1$ ) of liquid

128 cultures grown overnight in LB medium was added to $100 \mu \mathrm{l}$ of K10T-1 medium in the microtiter

129 plate, and statically incubated at $30^{\circ} \mathrm{C}$ for $6 \mathrm{hrs}$. After incubation, the liquid cultures were

130 discarded, and the biofilm cells were stained with $0.1 \%$ (wt/vol) crystal violet (CV) in water.

131 Twenty minutes later, the microtiter plates were rinsed with water three times, and air dried.

132 Quantification of the biofilm cells was performed as previously described (Monds et al., 2007).

133 In brief, $150 \mu \mathrm{l}$ of $30 \%$ acetic acid (vol/vol) was added to the microtiter plate to solubilize the CV,

134 and $125 \mu \mathrm{l}$ of this solution was transferred into a flat-bottom microtiter plate. A microplate reader

135 Vmax (Molecular Devices) was used to read the absorbance at 550nm.

136 Constructs for clean deletion and complementation. The pMQ30 and pMQ72 vectors

137 were used for clean deletion and complementation, respectively. Clean deletion mutants were

138 prepared as follows. A $\sim 1 \mathrm{kbp}$ pair of PCR fragments was amplified from upstream and

139 downstream of the target genes. The fragments were cloned into pMQ30 in parallel. Deletion

140 constructs were transferred into $P$. fluorescens by conjugation, and transconjugates were selected

141 on LB plates containing $\mathrm{Gm}$ and $\mathrm{Cm}$. After confirming the single-crossover events by PCR, the

142 strains were cultured overnight in the absence of any antibiotics and then spread on LB medium

143 agar plate containing 5\% (wt/vol) sucrose to facilitate the second crossover recombination. The 
144 removals of the target genes were verified by PCR, and all mutants resulted in the reduced 145 biofilm in the biofilm formation assay. The pMQ72 vectors for complementation were designed 146 to possess the ribosomal binding site (RBS) for LapD (Newell, Monds \& O'Toole, 2009) and the 147 N-terminal $6 \times$ histidine $(6 \times$ His $)$ tag. Success in construction was confirmed by DNA sequencing. 148 The pMQ72 vectors were introduced into the P. fluorescens strains by electroporation.

149 Measurement of numbers of planktonic and biofilm cells. Number of cells in the 150 supernatants and biofilms were determined by a serial dilution method. After incubation for 151 biofilm assay, the supernatants were removed, and the wells were washed twice with $100 \mu l$ of 152 PBS buffer consisting of $10 \mathrm{mM} \mathrm{Na}_{2} \mathrm{HPO}_{4}, 2 \mathrm{mM} \mathrm{KH}_{2} \mathrm{PO}_{4}, 137 \mathrm{mM} \mathrm{NaCl}$, and $2.7 \mathrm{mM} \mathrm{KCl}$. An 153 aliquot $(100 \mu \mathrm{l})$ of the PBS buffer was added to the wells, and the biofilm cells were collected 154 using a disposal cotton swab (Shimada et al., 2012). The cotton swab was then put into an 155 Eppendorf tube, and the well was washed twice with $100 \mu 1$ of the PBS buffer. The washed 156 buffers were transferred into the Eppendorf tube each time. After adjusted to $1 \mathrm{~mL}$, the solution 157 was vigorously vortexed and used for the serial dilution to determine the numbers of the cells. 158 Typically, $10 \mu \mathrm{l}$ of the diluted solutions were put onto the solidified LB medium plate, and 159 incubated at $30^{\circ} \mathrm{C}$ until the colonies became large enough to count. The experiments were 160 replicated four times for each sample, and the numbers of the colonies on the LB plates were 161 counted to calculate averages and standard deviations. This procedure was performed at least 162 twice. Successful removal of the biofilm cells from the wells was confirmed by staining the 163 microtiter plates with $\mathrm{CV}$, where the residual stain was negligible for all cases.

164 Measurement of the total cellular concentrations of ATP. The cellular concentrations of 165 ATP for the planktonic and biofilm cells for the WT and mutants were measured using a 166 BacTiter-Glo Microbial Cell Viability Assay kit from Promega (Madison, USA), and were 167 expressed in relative light unit (RLU). The measurements for RLU were performed using a 168 Lumitester C-100 from Kikkoman (Japan). The planktonic and biofilm cells derived from the 169 biofilm assay were used for the measurements, as described above. For the measurements of the 170 planktonic cells, $100 \mu \mathrm{l}$ of the supernatants were mixed with $100 \mu 1$ of the BacTiter-Glo reagent. 171 After incubated at $\sim 5 \mathrm{~min}$, RLUs were measured. For biofilm cells, the attached cells were finally 172 diluted to 10-fold with the PBS buffer. Each $100 \mu 1$ was used for the RLU measurements. The 173 experiments were repeated four times for each sample, and the averages and standard deviations 174 were calculated. The Student's t-test was performed to see difference between WT and the 175 mutants. 
Measurement of cell surface LapA by dot-blot. Bacteria were grown statically in K10T-1

177

178

179

180

181

182

183

184

185

186

187

188

189

190

191

192

193

194

195

196

197

198

199

200

201

202

203

204

205

206

207

with or without $0.2 \mathrm{mM}$ adenine for the indicated incubation period, or shaking at $250 \mathrm{rpm}$ as indicated. Then cells were harvested and resuspended at a cell density of $\mathrm{OD}_{600}=0.25$ in PBS, and spotted in $5 \mu \mathrm{l}$ aliquots onto nitrocellulose membrane filters. After drying, blots were blocked for 2 hours with a 1\% solution of Bovine serum albumin (BSA) in Tris-Buffered Saline (pH 7.4) containing $0.1 \%$ Tween (TBST). Then blots were probed for the HA-tagged LapA protein with anti-HA antibody (Fisher Scientific, RB1438P0) at a concentration of $2 \mu \mathrm{g} / \mathrm{ml}$ in TBST $1 \%$ BSA. After 3 washes of 10 minutes each in TBST, blots were probed with a horse radish peroxidaseconjugated secondary antibody (Fisher Scientific, PI-31460) at a concentration of 80ng/ml, then rinsed, imaged and quantified as previously described (Newell et. al, 2009).

Scanning electron microscopy. Scanning electron micrographs were obtained for the biofilms formed inside the 96-well microtiter plate, followed by platinum ion sputtering (MSP1S magnetron sputter, Vacuum Device Inc., Japan). A desktop scanning electron microscopy Phenom ProX (Phenom World, The Netherlands) was employed with the accelerating voltage of $10 \mathrm{kV}$. To obtain information on cell size, the electron micrographs were analyzed using Phenom ParticleMetric software (Phenom World, The Netherlands). From each micrograph, single cells were automatically identified using the following parameters: minimum contrast, 0.50 ; merge shared borders, 0.25 0.35; conductance, 0.25 0.30; minimum detection size (\%), 0.50. After manually removing the non-cell-derived small particles and the aggregated cells from the data collections, the histograms and parameters such as circumscribed circle diameter and circle equivalent diameter were automatically obtained. Five to eighteen SEM images were used to collect more than 1,000 of the circumscribed circle diameters for each analysis, which depended on the numbers of the cells in each micrograph. Analyses were performed at least three times for each sample.

Laser diffraction particle analysis. Size distributions of the planktonic and biofilm cells were measured using a laser diffraction particle analyzer LA-960 (Horiba, Japan). Particle size analysis was performed with the software equipped with the device. In this analysis, the software calculates the size of particles based on the Mie theory, which assumes the particles to be spherical (Eshel et al., 2004). Because the mutants did not grow well in K10T-1 medium, it was anticipated that the amounts of the cells were not enough for the measurements. We therefore used a prototype $200 \mu \mathrm{l}$ volume microcell for the measurements, which is not commercially available currently. The biofilm formation assays were performed with $10 \mathrm{ml}$ of K10T-1 in a 
208 standard $50 \mathrm{ml}$ centrifugal tube made of polypropylene. After static incubation at $30^{\circ} \mathrm{C}$ for $6 \mathrm{~h}$, the 209 planktonic cells were collected by centrifugation $(7.4 \mathrm{k} \mathrm{rpm}$ for $2 \mathrm{~min}$ ), and the cell-free 210 supernatants were discarded so that the residual bacterial solutions were roughly $200 \mu 1$. The 211 bacterial cells were resuspended using a micropipette, and then placed into the microcell using a 212 disposable plastic syringe. For the measurement of biofilm cells, the $50 \mathrm{ml}$ tubes were washed 213 twice with the PBS buffer, and then $10 \mathrm{ml}$ of the fresh buffers were added. The biofilm cells 214 were dispersed using an ultrasound homogenizer VC-130 equipped with a $2 \mathrm{~mm}$ microtip (Sonics 215 \& Materials, Inc.). The amplitude was set to $20 \sim 25 \%$. The dispersed biofilm cells were collected 216 by centrifugation and used for the measurements as described above. The measurements were 217 repeated at least three times for each sample. 
219 Results

220 Mutants incapable of de novo purine biosynthesis show reduced biofilm formation, but are

221 rescued by addition of adenine and hypoxanthine. Our previous transposon mutagenesis study

222 indicated that the genes involved in the de novo purine nucleotide biosynthesis are required for

223 normal biofilm formation by P. fluorescens Pf0-1 (Newell et al., 2011b). To verify this, we

224 constructed the clean deletion mutants for eight genes among total of eleven genes located in the

225 pathway from PRPP to IMP, and examined their biofilm formation (Fig. 1A). Both PurN and

226 PurT catalyze the same enzymatic step from 5-phosphoribosylglycinamide (GAR) to 5'-

227 phosphoribosyl- $N$-formylglycinamide (FGAR). Since it was anticipated that the deletion of one

228 of the genes would be compensated by the other, we did not make mutants for these genes. For

229 the $\triangle p u r B$ mutant, we were unable to delete the gene despite repeated attempts. We therefore did

230 not investigate these three genes in this study. As shown in Fig. 1A, biofilm formation by the

231 mutants was reduced to less than half of that by WT, which confirms the transposon mutagenesis

232 study.

233 To test whether these mutations were sufficient to explain the decrease in biofilm, we 234 performed a complementation analysis, reintroducing each gene on a plasmid (Fig. 1B).

235 Sufficient restoration of the biofilm formation was observed for complemented strains of $\Delta p u r D$,

$236 \Delta p u r H, \Delta p u r L, \Delta p u r C$, and $\Delta p u r K$. The recovery of biofilm formation was partial for 237 complemented strains of $\triangle p u r M, \Delta p u r F$, and $\triangle p u r E$ (Fig. 1B). Instead, full recovery of biofilm 238 formation for these three mutants was achieved by the addition of adenine (final conc. $1 \mathrm{mM}$ ) to 239 the medium (Supporting Fig. S1).

240 We next examined the effect of adding purine bases to the growth medium on biofilm 241 formation by WT and three mutants, $\Delta p u r H, \Delta p u r C$, and $\Delta p u r K$, was examined (Fig. 1C). Due to 242 low solubility of xanthine in the medium, the concentration of the purines added to the K10T-1 243 medium were set to $0.2 \mathrm{mM}$ in this experiment. In the salvage pathway, adenine, guanine, 244 hypoxanthine and xanthine are converted to the corresponding nucleotides by adenine 245 phosphoribosyltransferase and hypoxanthine-guanine phosphoribosyltransferase and xanthine 246 phosphoribosyltransferase. In E. coli and Salmonella, it is known that addition of adenine, 247 hypoxanthine, and guanine to the medium recovers the growth of the purine auxotroph mutants 248 (Neuhard \& Nygaar, 1987). This is because these bacteria possess adenosine deaminase and 249 GMP reductase that convert adenosine and GMP to inosine and IMP, respectively, enabling the 250 bacteria to synthesize both AMP and GMP from these purine bases. 
251

252

253

254

255

256

257

258

259

260

261

262

263

264

265

266

267

268

269

270

271

272

273

274

275

276

277

278

279

280

281

282

The biofilm phenotypes shown in Fig. 1C indicated that only adenine and hypoxanthine were able to recover the same level of biofilm formation as that of WT. On the other hand, addition of guanine or uric acid did not affect biofilm formation. BLAST searches indicated that $P$. fluorescens Pf0-1 possesses a homologous gene (Pf101_0671) to the adenosine deaminase of E. coli with sequence identity of $28 \%$, but there was no hit for the GMP reductase, suggesting lack of the latter enzyme in this microorganism. Interestingly, xanthine and oxidized purines, isoguanine and 8-hydroxyguanine, slightly promoted biofilm formation by $\Delta p u r H$ and $\Delta p u r K$. Incorporation of xanthine into DNA and RNA was previously reported for E. coli mutants that cannot convert IMP to XMP or AMP (Pang et al., 2012). A similar mechanism may work for this case. In contrast, caffeine, theobromine and theophylline that are the methylated derivatives of xanthine were insensitive to the biofilm formation by WT, $\Delta p u r H$ and $\Delta p u r K$. Very minor inhibitory effect was found for $\Delta p u r C$ (Fig. 1C).

Growth of the surface-attached cells of the mutants became slower than WT. As shown in Fig. 1D, the biofilm formation by WT reached maximum at around 6hrs and then started to decline, which could be caused by dispersal of the cells due to nutrient deficiency (see Newell et al., 2011a). In contrast, the amounts of biofilms by the $\Delta p u r H, \Delta p u r C$, and $\triangle p u r K$ mutants reached maxima at around 9 12hrs (Fig. 1D), indicating that the growth of the surface-attached cells of the mutants were slower than WT.

Mutations cause growth defect in the planktonic cells. One of the reasons for the reduced biofilm formation by the mutants could be a growth defect in the medium used in the assay, K10T-1. The supernatant for WT in the biofilm formation assay gradually became cloudy due to the growth of the cells. On the other hand, those for the mutants were clear and did not become turbid. To compare the growth of the cells during planktonic culture in this medium, changes in the absorbance at $590 \mathrm{~nm}\left(\mathrm{OD}_{590}\right)$ were monitored for WT and the above three mutants $(\Delta p u r H$, $\triangle p u r C$, and $\triangle p u r K$ ) (Fig. 2A). It was apparent that the $\mathrm{OD}_{590}$ for mutants did not change during $6 \mathrm{hrs}$, indicating that the mutants were not able to grow in K10T-1 medium. Addition of adenine into the medium rescued mutant growth, as the $\mathrm{OD}_{590}$ started to rise sharply at around $4 \mathrm{hrs}$, as observed for WT (Fig 2B). This result is consistent with the recovery of the biofilm formation in the presence of adenine, as shown in Supporting Fig. S1. It therefore seems likely that reduced biofilm formation by mutants in de novo purine biosynthesis pathway is due, in part, to the inability to grow in K10T-1 medium.

Mutations lead to purine deprivation in both planktonic and biofilm cells. Since the 
283 mutants lack one of the genes in the biosynthesis pathway in the IMP synthesis, and demonstrate 284 a growth defect in K10T-1 medium in the absence of purine supplementation, we predicted that 285 the cellular concentration of purine nucleotides should be lower in the mutants than in the WT in 286 K10T-1. Using the luciferase-based ATP assay we measured the relative concentrations of ATP 287 in both planktonic and biofilm cells. In Fig. 2C, the black and grey bars indicate ATP contents 288 for the planktonic and biofilm cells, respectively. The total ATP contents in the planktonic cells 289 of the mutants were almost 20-times lower than that of the WT cells. Similarly, those in the 290 surface-attached cells of the mutants were roughly 10-times lower than that of WT. These results 291 indicate that the cellular concentrations of purines are likely to be reduced in the mutant cells.

Purine biosynthesis mutations alter the proportion of attached verses planktonic cells

in static culture. Although biofilm formation by the mutants was reduced to less than half of that by WT (Fig. 1A), it conversely suggests that certain numbers of mutant cells were attached to the surface as biofilms. To examine this more closely, the numbers of cells in the planktonic and attached populations were determined (Fig. 2D). It is known that LB medium contains substantial amounts of nucleic acids derived from yeast extract. Therefore, it is not surprising that the mutants grew well in LB medium, which was used to culture the inoculum for the biofilm assay (black bars in Fig. 2D).

Comparing dark and light grey bars in Fig. 2D provides information on the proportion of biofilm cells versus planktonic cells, respectively. The fraction of the total cells that were attached to the surface was larger for the $\Delta p u r C(\sim 69 \%), \Delta p u r K(\sim 75 \%)$ and $\Delta p u r H(\sim 48 \%)$ mutants, compared to that for WT $(\sim 16 \%)$. These data seem to indicate that the mutants exhibit a growth defect in planktonic culture in K10T-1, and are consistent with the growth curves shown in Fig. 2A. They are also consistent with the survival of cells under purine deprivation in the biofilm mode of growth.

Secondly, it should be noted that the cell numbers of the biofilms for the $\Delta p u r H$ and $\Delta p u r K$ 308 mutants were almost the same as that of WT. Remember that the CV staining of $\Delta p u r H$ mutant biofilms was reduced to $\sim 40 \%$ of the WT (Fig. 1A). One explanation for the reduction in biofilm biomass by the $\Delta p u r H$ mutant could be a reduction in cell size of the $\Delta p u r H$ mutant compared to that of WT. In contrast, the cell number from the biofilm of the $\triangle p u r C$ mutant was one-order of magnitude lower than the others. Therefore, reduction in cell number should also be considered

313 as a contributor to reduced biofilm formation by the mutants. Altogether, these observations 314 provided a hypothesis that the reduction in biofilms by the mutants is originated from the 
315 reduction(s) in cell sizes and/or cell numbers for the biofilm cells.

316 Purine deprivation reduces amount of the surface adhesin, LapA in the planktonic cells.

317 Biofilm formation by $P$. fluorescens Pf0-1 requires initial attachment via the cell surface adhesin,

318 LapA (Hinsa et al., 2003; Boyd et al., 2014). The amount of this protein on the cell surface is

319 controlled by internal signals via the binding of c-di-GMP to LapD (Monds et al., 2007; Newell

320 et al., 2009). We next addressed whether the pur mutants were capable of attachment via LapA,

321 despite showing reduced ATP levels which could influence cellular GTP and c-di-GMP

322 concentrations. To test LapA levels during initial attachment, cells were incubated statically in

323 K10T-1 medium for 1 hour with or without adenine. Next, cells were harvested and probed for

324 cell surface LapA by dot-blot. We found the LapA level was significantly reduced in the $\Delta p u r H$,

$325 \Delta$ purC and $\Delta p u r K$ mutants compared to WT $(P<0.001$, Wilcoxon Sum Rank Test $)$, however the

326 reduction was not more than $40 \%$ (Fig. 2E). By comparison, deletion of lapD has been shown to

327 reduce cell-surface LapA by $>90 \%$ (Newell et al., 2009). Addition of adenine to the medium

328 significantly increased LapA localization to the cell surface in all of the mutants $(P<0.001)$, but

329 had no impact on LapA localization in the WT (Fig. 2E). Only in the case of the $\Delta p u r H$ mutant

330 did adenine supplementation restore the LapA level to one indistinguishable from WT $(P>0.5)$,

331 while the $\triangle p u r C$ and $\triangle p u r K$ mutants were partially restored to $88 \%$ and $92 \%$ of WT, respectively.

332

333

We hypothesized that further reduction in cell surface LapA might be observed for the mutants after prolonged incubation in the absence of adenine. To test this, cells were incubated in K10T-1 for 9 hours under aerated conditions. These cells showed a greater reduction in cell surface LapA relative to WT than that seen during the initial attachment phase $(\Delta p u r H 20 \%$, $\Delta p u r C 13 \%$, and $\Delta$ purK $14 \%$ of WT level; Fig. 2F). Altogether, the LapA quantification results are consistent with the pur mutants attaching to surfaces via the LapA adhesin, albeit at a lower level than that of WT, and indicate that purine deprivation reduces the amount of LapA on the cell surface of planktonic cells.

Cell size is altered by purine deprivation, and by surface attachment independent of purine deprivation. From the cell counting experiments (Fig. 2D), the numbers of the surfaceattached cells of the $\Delta p u r H$ and $\Delta p u r K$ mutants were not significantly different from that of WT, while the amounts of biofilms by the mutants were less than half of WT (Fig. 1A). To explain the reduction in the biofilm formation by the mutants, we hypothesized that the mutant cells in 
347 cultured in K10T-1 were obtained (Fig. 3A). These data indicated that the amounts of the mutant

348 cells in each view field of the SEM image were less than that of WT, which seems contradict to

349 the results in the cell numbers (Fig. 2D). However, this is not unexpected because the mutant

350 cells are seen at wider region in the wells of the microplate than WT whose biofilm cells are

351 observed only at the air-liquid interface (Fig. 1A). To clarify the difference in the cell sizes

352 between WT and the mutants, the SEM images were analyzed by measuring the circumscribed

353 circle diameter of individual cells as an approximation for the length of the cell (described

354 further in Materials \& Methods). We used the Wilcoxon Sum Rank test to compare the medians

355 (Table 1). The medians for the circumscribed circle diameter for the $\Delta p u r H, \Delta p u r C$, and $\Delta p u r K$

356 mutants were $1.31,1.35$, and $1.43 \mu \mathrm{m}$, respectively, and became significantly smaller than WT

$357(1.87 \mu \mathrm{m})$ by $25 \sim 30 \%$.

358 As the impaired biofilm formation by the mutants was restored by the supplementation of

359 adenine (Fig. 1C), we expected that the cell sizes for the mutants would return to the equivalent

360 size of WT. Figure 3B shows the electron micrographs and histograms obtained for the adenine-

361 supplemented biofilm cells of WT and the mutants. Against our expectation, the WT cells

362 showed a slightly smaller size $(1.82 \mu \mathrm{m})$ compared to that $(1.87 \mu \mathrm{m})$ in K10T-1 medium. Growth

363 inhibition of $E$. coli by adenine supplementation has been known, which has been supposed to be

364 attributed to inhibition of the de novo biosynthesis of pyrimidine nucleotides (Hosono \& Kuno,

365 1974) or depletion of cellular concentrations of GTP (Levine \& Taylor, 1982) and PRPP

366 (Shimosaka et al., 1984). Similar mechanisms may work for the WT strain of P. fluorescens Pf0-

367 1, resulting in the observed slight shrinkage of the biofilm cells in the presence of adenine. The

368 circumscribed circle diameters of the $\Delta p u r H, \Delta p u r C$, and $\Delta p u r K$ mutants when adenine was

369 added to K10T-1 were 1.59, 1.70, and $1.63 \mu \mathrm{m}$, respectively. This represents a significant

370 increase in mutant cell size due to the addition of adenine, ranging between 14-26\%, suggesting

371 that adenine supplementation can partially rescue the defect in cell size shown by the mutants

372 (Table 1).

373 As stated above, biofilm formations by WT and the mutants showed time-dependent changes

374 (Fig. 1D). At later than 6hrs, the amounts of biofilms by the mutants gradually increased and

375 came close to that of WT. These observations suggest that the biofilm cells of the WT become

376 smaller while the mutant cells become larger due to the cell growth. To elucidate this point, we

377 obtained the SEM images for the biofilm cells at 9hrs, and the median sizes of the surface-

378 attached cells were determined (Fig. 3C and Table 1). 
WT cells became significantly smaller $(1.44 \mu \mathrm{m})$ than that at $6 \mathrm{hrs}(1.87 \mu \mathrm{m} ; P<0.01)$, at

380

381

382

383

384

385

386

387

388

389

390

391

392

393

394

395

396

397

398

399

400

401

402

403

404

405

406

407

408

409

410 longer incubation time. In contrast, the median sizes for the $\triangle p u r H, \triangle p u r C$, and $\triangle p u r K$ mutants were 1.54, 1.42, and $1.61 \mu \mathrm{m}$, respectively (Fig. 3C and Table 1), all significantly larger than those at $6 \mathrm{hrs}$ but still smaller than the original size (WT at $6 \mathrm{hrs}, 1.87 \mu \mathrm{m}$; see $P$ values in Table 1). The increases in cell sizes (Fig. 3C) and amounts of biofilms (Fig. 1D) observed for the mutants may indicate gradual cell growth of the surface-attached cells despite purine limitation.

Biofilm cells are smaller than planktonic cells. As revealed by the SEM analyses, the biofilm cells of the mutants possess smaller cell sizes than WT. The observation seems to be similar to that happens when bacteria are placed under condition like carbon starvation (Östling et al., 1993) or undergo what are typically called reductive divisions (Roszak \& Colwell, 1987; Nyström, 2004). In the latter case, cell number increases without significant increase in biomass, which accompanies decrease in the cell size (Roszak \& Colwell, 1987; Nyström, 2004). The reason for our observation may be ascribed to survival of the mutants on the surface under the purine limitation. This is an interesting hypothesis, but has not been confirmed yet. One way to test this is to know the sizes of the planktonic cells and compare those of the biofilm cells.

To get insights into the cell sizes for the planktonic cells, the size distributions for the planktonic cells were measured using a laser diffraction particle analyzer (Fig. 4). We compared the mode diameters that are the highest peak of the frequency distribution and represent the most commonly found cell (particle) sizes in the sample. To show distribution width for each analysis, D10, D50 (median), and D90 are shown in Table 2.

Shown in Fig. 4A are the frequency distributions for the planktonic cells of WT cultured in K10T-1 (solid line) and K10T-1+adenine (dashed line). Note that the solid line almost overlapped the dashed line, indicating little change in the size of planktonic cells with or without adenine. The mode diameter for WT cells grown in both conditions was $1.86 \mu \mathrm{m}$. Biofilm cells were also analyzed after removal from the surface, which is shown in the dotted line in Fig. 4A. The mode diameter of WT cells growing in a biofilm was significantly smaller than that of the planktonic cells in K10T-1 $\left(1.62 \mu \mathrm{m}\right.$ vs. $1.86 \mu \mathrm{m}$; t-test, $\left.P<10^{-10}\right)$, suggesting that the change in life style from the planktonic to the surface-attached makes the cell size smaller for the WT cells (Table 2).

A reduction in the sizes of biofilm cells compared to planktonic cells was also observed for the mutants (Table 2). The mode diameter measurements of the mutant planktonic cells in K10T1 medium ranged from $1.86 \mu \mathrm{m}$ to $1.94 \mu \mathrm{m}$ but were not significantly different from WT $(P<$ 
4110.05 ; t-test). In contrast, the mode diameters of the mutant biofilm cells were significantly 412 smaller than planktonic or WT biofilm cells. The mode diameters were $1.08 \mu \mathrm{m}$ for the $\Delta p u r H$ 413 and $\triangle p u r C$ mutants and $1.13 \mu \mathrm{m}$ for the $\Delta p u r K$ mutant, which corresponds to $30 \sim 33 \%$ reduction 414 in size compared to WT biofilm cells $(P<0.001$, t-test $)$ and a $42-44 \%$ reduction compared to the 415 planktonic cells of each strain $(P<0.002$, t-test $)$. These results are consistent with the SEM 416 analyses that indicated $25 \sim 30 \%$ of reductions in the circumscribed circle diameters for mutant 417 biofilm cells compared to WT.

418 Supplementation of adenine had minimal effects on the size planktonic mutant cells, 419 indicating that the planktonic cells of the mutants maintain the same cell size irrespective of 420 purine levels in the growth medium (Table 2; difference from same strain without adenine not 421 significant by t-test, $P>0.05$ ). In summary, the laser diffraction particle analyzer data revealed 422 significant differences between the size of planktonic and biofilm cells for the pur mutants and 423 the WT. They also corroborate the observation by SEM that the surface-attached mutant cells 424 have a smaller cell size than that of WT. Taken together with our analysis of CFU in the 425 planktonic vs. attached phases of static cultures (Fig. 2D), these data suggest major differences in 426 the physiology of attached cells under purine limitation compared to their free-floating 427 counterparts. 
429

430

431

432

433

434

435

436

437

438

439

440

441

442

443

444

445

446

447

448

449

450

451

452

453

454

455

456

457

458

459

460

\section{Discussion}

In this study, we sought to determine the basis for the defects in the biofilm formation by the purine auxotrophic mutants of $P$. fluorescens Pf0-1, in which one of the genes involved in the de novo purine biosynthesis pathway to IMP was disrupted. We found that the attached biomass in the mutant biofilms was less than half of that in WT biofilms in K10T-1 medium (Fig. 1A). As this biosynthesis pathway is essential for most of bacteria, many studies have shown that the disruption of the genes in this biosynthesis pathway also impact virulence, biofilm formation and symbiosis. Our results confirm that the purine auxotrophic mutants of $P$. fluorescens Pf0-1 show a reduced biofilm phenotype and provide details as to the mechanisms involved.

Synthesizing our results, we propose that the biofilm phenotype of the pur mutants is influenced by several factors. First, the mutations resulted in modest but significant reductions in cell surface LapA (Fig. 2E), which likely reduces the number of cells that initially attach to the surface. This is consistent with the lower density of attached cells observed by SEM (Fig. 3 and Table 1). Second, as the exogenous purine supply is exhausted during static growth in K10T-1 the proliferation of the mutants is likely slowed due to purine limitation; this judgment is based on planktonic growth and biofilm cell counts (Fig 2A and 2D). The growth defect observed in the absence of purine supplementation is one major contributing factor in the decrease in biofilm formation.

A third factor influencing the biofilm phenotype of the pur mutants is changes in cell size. Under purine limitation, attached cells became reduced in size, about $25-30 \%$ smaller than WT by SEM (Fig. 3). This is consistent with the results from the laser diffraction particle analyses which also indicated a 30-33\% size reduction for the mutants (Fig. 4 and Table 2), while the two methods utilize different theories to obtain the cell sizes. The attached mutant cells were significantly smaller than attached WT cells at 6 hours when the greatest difference in attached biomass is observed by the CV assay (Fig. 1D). The amount of attached biomass and biofilm cell sizes for the WT and mutants converge at 9 hours, likely due to the gradual elongation of mutant cells and potentially shortening of WT cells, which we predict experience some nutrient limitation by this time (Fig. 1D and 3).

One interesting finding of this study is the difference between attached and planktonic mutant cells. These differences are evident in the change in cell size upon purine limitation (Fig. 4), as well as in the larger number of viable cells recovered from the biofilm compared to the planktonic population for $\triangle p u r C$ and $\triangle p u r K$ (Fig. 2D). Additionally, the size of biofilm cells 
461 increased in response to adenine supplementation, while the planktonic cells did not (Fig. 3 vs. 462 Fig. 4). Combined with the modest increase in mutant biofilm cell size between 6 and 9 hours, 463 and ATP measurements (Fig. 2C), these data argue that purine-limited biofilm cells are 464 metabolically active.

465 Although the result in Fig. 2D indicated that the number of the biofilm cells was decreased 466 one-order of magnitude for the $\triangle p u r C$ mutant, the ATP assay revealed that the total amount of 467 ATP for this mutant was similar to those of the $\Delta p u r H$ and $\Delta p u r K$ mutants (Fig. 2C). These 468 results may indicate that there are some problems in recovery process for the $\Delta p u r C$ mutant after 469 plating on a LB medium plate in the cell counting experiments. In other words, the actual 470 number for the biofilm cells for the $\triangle p u r C$ mutant may be higher than the value indicated by 471 plate counts (Fig. 2D) and thus closer to those of the other mutants.

472 The laser diffraction study further revealed that the biofilm cells of WT are smaller than 473 those in the planktonic phase of the same medium (Fig. 4 and Table 2). A similar observation 474 was reported for Staphylococcus aureus, in which differences in total cellular proteins and 475 respiratory activity between surface-attached and planktonic cells were observed (Williams et al., 476 1999). Therefore, our observation suggests that some metabolic differences exist between the 477 two states. In addition, the cells sizes of WT biofilms in the presence of adenine are smaller than 478 those in its absence (Fig. 3B and Table 1). For the purine auxotrophic mutants, the salvage 479 pathway is the sole way to synthesize the purine nucleotides. However, for WT, the excess 480 purine base in the medium not only suppresses the de novo purine biosynthesis pathway 481 (Houlberg \& Jensen, 1983) but also may influence other metabolic pathways, leading to the 482 observed shrinkage of the surface-attached cells. Further study using metabolomic and/or gene 483 expression analyses is desired to identify the mechanism behind these changes.

484 In contrast to the surface-attached cells, the planktonic cells of the mutants remain the same 485 cell size as WT irrespective of adenine supplementation (Fig. 4 and Table 2). As shown in Fig. $4862 \mathrm{D}$, the numbers of the planktonic cells for the mutants were less than the inoculums, indicating 487 cell death occurs for some planktonic cells that fail to adapt to the purine deficiency. The 488 behavior of the planktonic cells of the mutants is different from those of Vibrio sp. and P. putida 489 KT2442, in which the cell size reduction occurred for the planktonic cells soon after they were 490 placed under carbon limitation (Amy \& Morita, 1983; Östling et al., 1993; Givskov et al., 1994).

491 This is an interesting observation as it indicates the surface-attached forms of the mutants are 492 more suitable for survival rather than the planktonic ones under the purine shortage. 


\section{Conclusions}

495 This study examined purine auxotrophic mutants of P. fluorescens Pf0-1 to elucidate the 496 basis of the defects they display in biofilm formation. We found significantly more viable mutant 497 cells attached to the surface than in the planktonic phase, indicating that the surface-attached 498 mode of growth is suitable for survival of the mutants under the purine shortage. ATP 499 measurements and the observed increases in cell size between 6 and 9 hours also suggest that 500 mutant biofilm cells are metabolically active. Using SEM and a laser diffraction particle analyzer, 501 we demonstrated that the surface-attached mutant cells have smaller sizes than WT and that the 502 surface-attached WT cells were smaller than the planktonic ones. The latter observation indicates 503 that some modulation of cell size is a natural response of $P$. fluorescens to the biofilm 504 environment. The reduction in the cell numbers and sizes in mutant biofilms could be main 505 factors to explain the reduced biofilm formation by the mutants. The data presented here 506 provides a new view on the relationship between purine deficiency and biofilm formation.

509 Abbreviations

510 CV crystal violet

511 SEM scanning electron microscopy

512

513 


\section{Ackowledgements}

515 We thank Shingo Shimoyama, Shoko Wada, and Atsumi Ozaki (Jasco International Co., Ltd.,

516 Japan) for their assistance in obtaining and analyzing the scanning electron micrographs. We are

517 grateful to Kyoko Mitsunari and Kazuhiro Yoshida (Horiba Ltd., Japan) for the measurements

518 using a laser diffraction particle analyzer. We also thank Dr. George O'Toole and two 519 anonymous reviewers for critical reading of the manuscript.

520 
521 References

522 Amy PS, Morita RY. 1983. Starvation-survival patterns of sixteen freshly isolated open-ocean

523 bacteria. Applied and Environmental Microbiology 45:1109-1115.

524

525 An R, Grewal PS. 2011. purL gene expression affects biofilm formation and symbiotic 526 persistence of Photorhabdus temperata in the nematode Heterorhabditis bacteriophora. 527 Microbiology 157:2595-2603. DOI: 10.1099/mic.0.048959-0.

528

529

Bacon GA, Burrows TW, Yates M. 1951. The effects of biochemical mutation on the virulence 530 of Bacterium typhosum: The loss of virulence of certain mutants. British Journal of Experimental 531 Pathology 32:85-96.

532

Boyd CD, Smith TJ, El-Kirat-Chatel S, Newell PD, Dufrêne YF, O’Toole GA. 2014. Structural 534 features of the Pseudomonas fluorescens biofilm adhesion LapA required for LapG-dependent 535 cleavage, biofilm formation, and cell surface localization. Journal of Bacteriology 196:2775536 2788. DOI: 10.1128/JB.01629-14.

537

Christen, M, Christen B, Folcher M, Schauerte A, Jenal U. 2005. Identification and characterization of a cyclic di-GMP-specific phosphodiesterase and its allosteric control by GTP. Journal of Biological Chemistry 280:30829-30837. DOI: 10.1074/jbc.M504429200.

542 Eshel G, Levy GJ, Mingelgrin U, Singer MJ. 2004. Critical evaluation of use of laser diffraction 543 for particle-size distribution analysis. Soil Science Society of America Journal 68:736-743. 544 DOI: $10.2136 /$ sssaj2004.0736.

546 Ge X, Kitten T, Chen Z, Lee SP, Munro CL, Xu P. 2008. Identification of Streptococcus 547 sanguinis genes required for biofilm formation and examination of their role in endocarditis 548 virulence. Infection and Immunity 76:2551-2559. DOI: 10.1128/IAI.00338-08.

550 Gerber ED, Hackett AJ, Franklin R. 1952. The virulence of biochemical mutants of Klebsiella 551 pneumoniae. Genetics 38:693-697. 
553 Givskov M, Eberl L, Møller S, Poulsen LK, Molin S. 1994. Responses to nutrient starvation in 554 Pseudomonas putida KT2442: Analysis of general cross-protection, cell shape, and 555 macromolecular content. Journal of Bacteriology 176:7-14.

556

557 Haas D, Defago G. 2005. Biological control of soil-borne pathogens by fluorescent 558 pseudomonads. Nature Reviews Microbiology 3:307-319. DOI: 10.1038/nrmicro1129.

559

560 Han SH, Anderson AJ, Yang KY, Cho BH, Kim KY, Lee MC, Kim YH, Kim YC. 2006.

561 Multiple determinatns influence root colonization and induction of induced systemic resistance

562 by Pseudomonas chlororaphis O6. Molecular Plant Pathology 7:463-472. DOI: 10.1111/J.1364563 3703.2006.00352.X.

564

565 Hinsa SM, Espinosa-Urgel M, Ramos JL, O’Toole GA. 2003. Transition from reversible to 566 irreversible attachment during biofilm formation by Pseudomonas fluorescens WCS365 requires 567 an ABC transporter and a large secreted protein. Molecular Microbiology 49:905-918. DOI: 568 10.1046/j.1365-2958.2003.03615.x.

569

570

Hosono R \& Kuno S. 1974. Mechanism of inhibition of bacterial growth by adenine. Journal of 571 Biochemistry 75:215-220.

572

573 Houlberg U, Jensen KF. 1983. Role of hypoxanthine and guanine in regulation of Salmonella 574 typhimurium pur gene expression. Journal of Bacteriology 153:837-845.

575

576

Jenkins A, Cote C, Twenhafel N, Merkel T, Bozue J, Welkos S. 2011. Role of purine

577 biosynthesis in Bacillus anthracis pathogenesis and virulence. Infection and Immunity 79:153166. DOI: 10.1128/IAI.00925-10.

579

580

Kim JK, Jang HA, Won YJ, Kikuchi Y, Han SH, Kim C-H, Nikoh N, Fukatsu T, Lee BL. 2014a.

581 Purine biosynthesis-deficient Burkholderia mutants are incapable of symbiotic accommodation

582 in the stinkbug. The ISME Journal 8:552-563. DOI: 10.1038/ismej.2013.168.

583

584 Kim JK, Kwon JY, Kim SK, Han SH, Won YJ, Lee JH, Kim C-H, Fukatsu T, Lee BL. 2014 b. 
585 Purine biosynthesis, biofilm formation, and persistence of an insect-microbe gut symbiosis.

586 Applied and Environmental Microbiology 80:4374-4382. DOI: 10.1128/AEM.00739-14.

587

588 Levine RA \& Taylor MW. 1982. Mechanism of adenine toxicity in Escherichia coli. Journal of 589 Bacteriology 149:923-930.

590

591 Monds RD, Newell PD, Gross RH, O’Toole GA. 2007. Phosphate-dependent modulation of c-di592 GMP levels regulates Pseudomonas fluorescens Pf0-1 biofilm formation by controlling secretion 593 of the adhesion LapA. Molecular Microbiology 63:659-679. DOI: 10.1111/j.1365594 2958.2006.05539.x.

595

596 Monds RD. Newell PD. Schwartzman JA. O’Toole GA. 2006. Conservation of the Pho regulon 597 in Pseudomonas fluorescens Pf0-1. Applied and Environmental Microbiology 72:19190-1924. 598 DOI: 10.1128/AEM.72.3.1910-1924.2006.

599

600 Monds RD, Newell PD, Wagner JC, Schwartzman JA, Lu W, Rabinowitz, JD, O’Toole GA. 601 2010. Di-adeonosine tetraphosphate (Ap4A) metabolism impacts biofilm formation by 602 Pseudomonas fluorescens via modulation of c-di-GMP-dependent pathway. Journal of 603 Bacteriology 192:3011-3023. DOI: 10.1128/JB.01571-09.

604

605 Neuhard J. and Nygaar P. 1987. Purines and Pyrimidines. In: Neidhardt FC, ed. Escherichia coli 606 and Salmonella typhimurium Cellular and molecular biology. Washington, D.C: American 607 Society for Microbiology. 445-473.

608

609 Newell PD, Boyd CD, Sondermann H, O’Toole GA. 2011a. A c-di-GMP effector system 610 controls cell adhesion by inside-out signaling and surface protein cleavage. PLoS Biology 611 9:e1000587. DOI: 10.1371/journal.pbio.1000587.

612

613 Newell PD, Monds RD, O'Toole GA. 2009. LapD is a bis-(3', 5')-cyclic GMP binding protein 614 that regulates surface attachment by Pseudomonas fluorescens Pf0-1. Proceedings of National 615 Academy of Sciences in the USA 106:3461-3466. DOI: 10.1073/pnas.0808933106. 616 
617 Newell PD, Yoshioka S, Hvorecny KL, Monds RD, O’Toole GA. 2011b. Systematic analysis of 618 diguanylate cyclases that promote biofilm formation by Pseudomonas fluorescens Pf0-1. Journal 619 of Bacteriology 193:4685-4698. DOI: 10.1128/JB.05483-11. 620

621 Nyström T. 2004. Stationary-phase physiology. Annual Review of Microbiology 58:161-181. 622 DOI: 10.1146/annurev.micro.58.030603.123818.

623

624 Östling J, Holmquist L, Flärdh K, Svenblad B, Jouper-Jaan Å, Kjelleberg S. 1993. Starvation and 625 Recovery of Vibrio. In: Kjelleberg S, ed. Starvation in Bacteria. New York: Plenum Press, 103626127.

627

628 Pang B, McFaline JL, Burgis NE, Dong M, Taghizadeh K, Sullivan MR, Elmquist CE, 629 Cunningham RP, Dedon PC. 2012. Defects in purine nucleotide metabolism lead to substantial 630 incorporation of xanthine and hypoxanthine into DNA and RNA. Proceedings of National 631 Academy of Sciences in the USA 109:2319-2324. DOI: 10.1073/pnas.1118455109.

632

633 Paul R, Weiser S, Amiot NC, Chan C, Schimer T, Giese B, Jenal U. 2004. Cell cycle-dependent 634 dynamic localization of a bacterial response regulator with a novel di-guanylate cyclase output 635 domain. Genes \& Development 18:715-727. DOI: 10.1101/gad.289504.

636

637 Pilatz S, Breitbach K, Hein N, Fehlhaber B, Schulze J, Brenneke B, Eberl L, Steinmetz I. 2006. 638 Identification of Burkholderia pseudomallei genes required for the intracellular life cycle and in 639 vivo virulence. Infection and Immunity 74:3576-3586. DOI: 10.1128/IAI.01262-05.

640

641 Polissi A, Pontiggia A, Feger G, Altieri M, Mottl H, Ferrari L, Simon D. 1998. Large-scale 642 identification of virulence genes from Streptococcus pneumoniae. Infection and Immunity $64366: 5620-5629$.

644

645 Roszak DB, Colwell RR. 1987. Survival strategies of bacteria in the natural environment. 646 Microbiological Reviews 51:365-379. 647

648 Ryan RP, Fouhy Y, Lucey JF, Crossman LC, Spiro S, He Y-W, Zhang L-H, Heeb S, Cámara M, 
649 Williams P, Dow JM. 2006. Cell-cell signaling in Xanthomonas campestris involves an HD650 GYP domain protein that functions in cyclic di-GMP turnover. Proceedings of National 651 Academy of Sciences in the USA 103:6712-6717. DOI: 10.1073/pnas.0600345103.

652

653 Ryjenkov DA, Tarutina M, Moskvin OV, Gomelsky M. 2005. Cyclic diguanylate is a ubiquitous 654 signaling molecule in bacteria: insight into biochemistry of the GGDEF protein domain. Journal 655 of Bacteriology 187:1792-1798. DOI: 10.1128/JB.187.5.1792-1798.2005. 656

657 Samant S, Lee H, Ghassemi M, Chen J, Cook JL, Mankin AS, Neyfakh AA. 2008. Nucleotide 658 biosynthesis is critical for growth of bacteria in human blood. PLoS Pathogens 4:e37. DOI: 659 10.1371/journal.ppat.0040037.

660

661 Schmidt, AJ, Ryjenkov DA, Gomelsky M. 2005. The ubiquitous protein domain EAL is a cyclic 662 diguanylate-specific phosphodiesterase: enzymatically active and inactive EAL domains. 663 Journal of Bacteriology 187:4774-4781. DOI: 10.1128/JB.187.14.4774-4781.2005.

664

665

666

667

Shanks RM, Caiazaa NC, Hinsa SM, Toutain CM, O’Toole GA. 2006. Saccharomyces cerevisiae-based molecular tool kit for manipulation of genes from gram-negative bacteria. Applied and Environmental Microbiology 72:5027-5036. DOI: 10.1128/AEM.00682-06.

Shimada K, Itoh Y, Washio K, Morikawa M. 2012. Efficacy of forming biofilms by naphthalene degrading Pseudomonas stutzeri T102 toward bioremediation technology and its molecular mechanisms. Chemosphere 87:226-233. DOI: 10.1016/j.chemosphere.2011.12.078.

672

673

Shimosaka M, Fukuda Y, Murata K, Kimura A. 1984. Purine-mediated growth inhibition caused by a purE mutation in Escherichia coli K-12. Journal of Bacteriology 160:1101-1104.

675

676

Straley AC, Harmon PA. 1984. Growth in mouse peritoneal macrophages of Yersinia pestis 678

679 Wang J, Mushegian A, Lory S, Jin S. 1996. Large-scale isolation of candidate virulence genes of 680 Pseumonas aeruginosa by in vivo selection. Proceedings of National Academy of Sciences in the 
681 USA 93:10434-10439.

682

683 Williams I, Paul F, Lloyd D, Jepras R, Critchley I, Newman M, Warrack J, Giokarini T, Hayes 684 AJ, Randerson PF, Venables WA. 1999. Flow cytometry and other techniques show that 685 Staphylococcus aureus undergoes significant physiological changes in the early stages of 686 surface-attached culture. Microbiology 145:1325-1333. 


\section{Figure 1 (on next page)}

Effect of the mutation of the genes in the de novo purine nucleotide biosynthesis on biofilm formation.

(A) A quantitative biofilm assay comparing WT and the clean deletion mutants. Data are the mean absorbance of dissolved crystal violet stained biomass at 550nm \pm standard deviations (SD) $(n=7)$. Representative images for the biofilms are shown above the graphs. The experiments were performed several times, and the representative one is shown. The asterisks $\left.{ }^{*}\right)$ indicate significant differences in absorbance at $550 \mathrm{~nm}\left(\mathrm{OD}_{550}\right)$ relative to that of WT ( $P<0.01$ in two-tailed Student's t-test assuming equal variance). (B) A quantitative biofilm assay to examine complementation of the genes for the clean deletion mutants (gray bars). The PMQ72 vector with each gene was introduced into the parent strain. The WT strain contained an empty PMQ72 vector. Arabinose was added to the medium at a concentration of $0.1 \%$ to induce the gene expression. Strains without the pMQ72 vector are shown in black bars as references. Data are the mean absorbance at 550nm \pm SD $(n=7)$. Representative images for the biofilms with the gene complementation are shown above the graphs. The asterisks $\left.{ }^{*}\right)$ indicate significant differences in absorbance at $550 \mathrm{~nm}\left(\mathrm{OD}_{550}\right)$ relative to that of WT $(P<0.01$ in two-tailed Student's t-test assuming equal variance). The experiments were performed several times, and one of the results is indicated. (C) A quantitative biofilm assay for WT (black), $\Delta p u r H$ (dark gray), $\Delta p u r C$ (gray), and $\Delta p u r K$ (light gray) in the presence of various purine bases. Data are the mean absorbance at 550nm $\pm \mathrm{SD}(n=8)$. Asterisks $(*)$ show a statistically significant difference in absorbance relative to the medium without exogenous purines ( $P<0.01$ in two-tailed Student's $t$-test assuming equal variance). Each purine base was added to the medium at concentration of $0.2 \mathrm{mM}$. The experiments were done at least twice. (D) Time-dependent changes in biofilm formations by the WT and mutants strains in K10T-1. Data at each time point are the mean absorbance at 550nm \pm SD $(n=7)$. The experiments were done duplicate, and one of which is shown. 
Figure 1

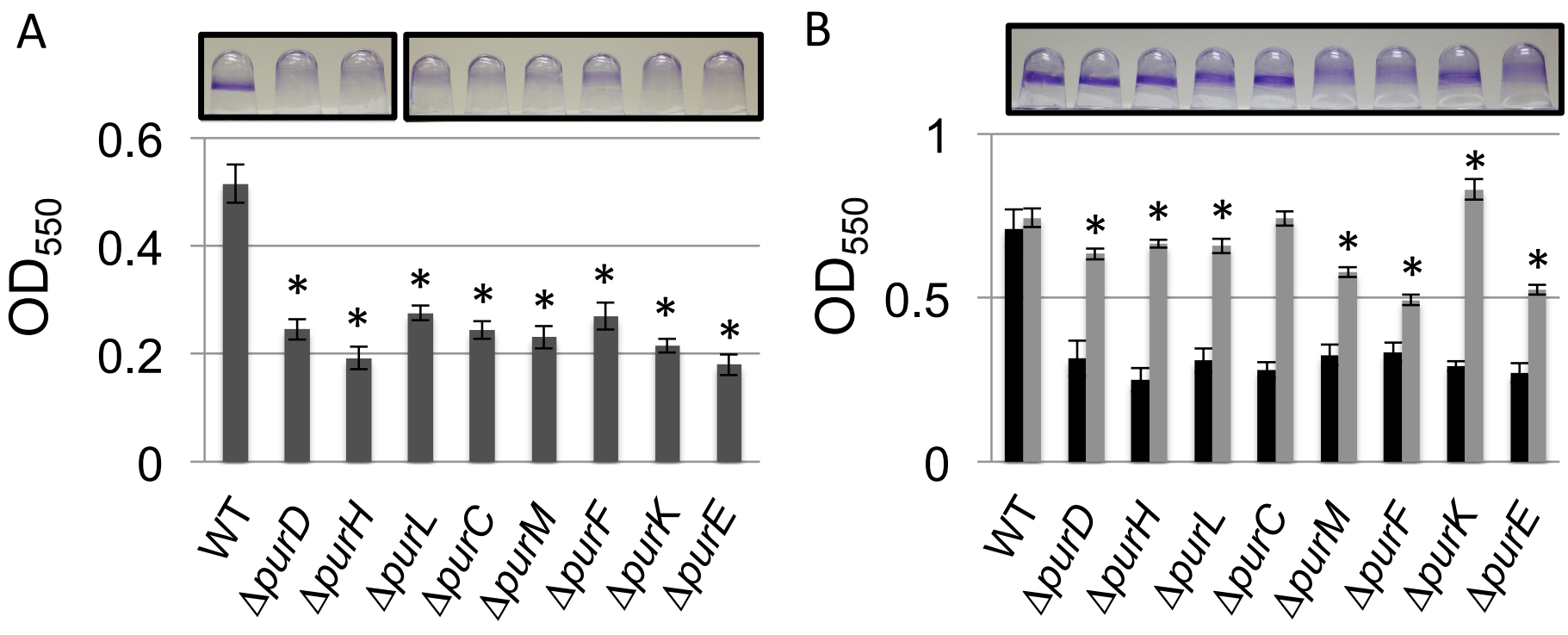

C 1
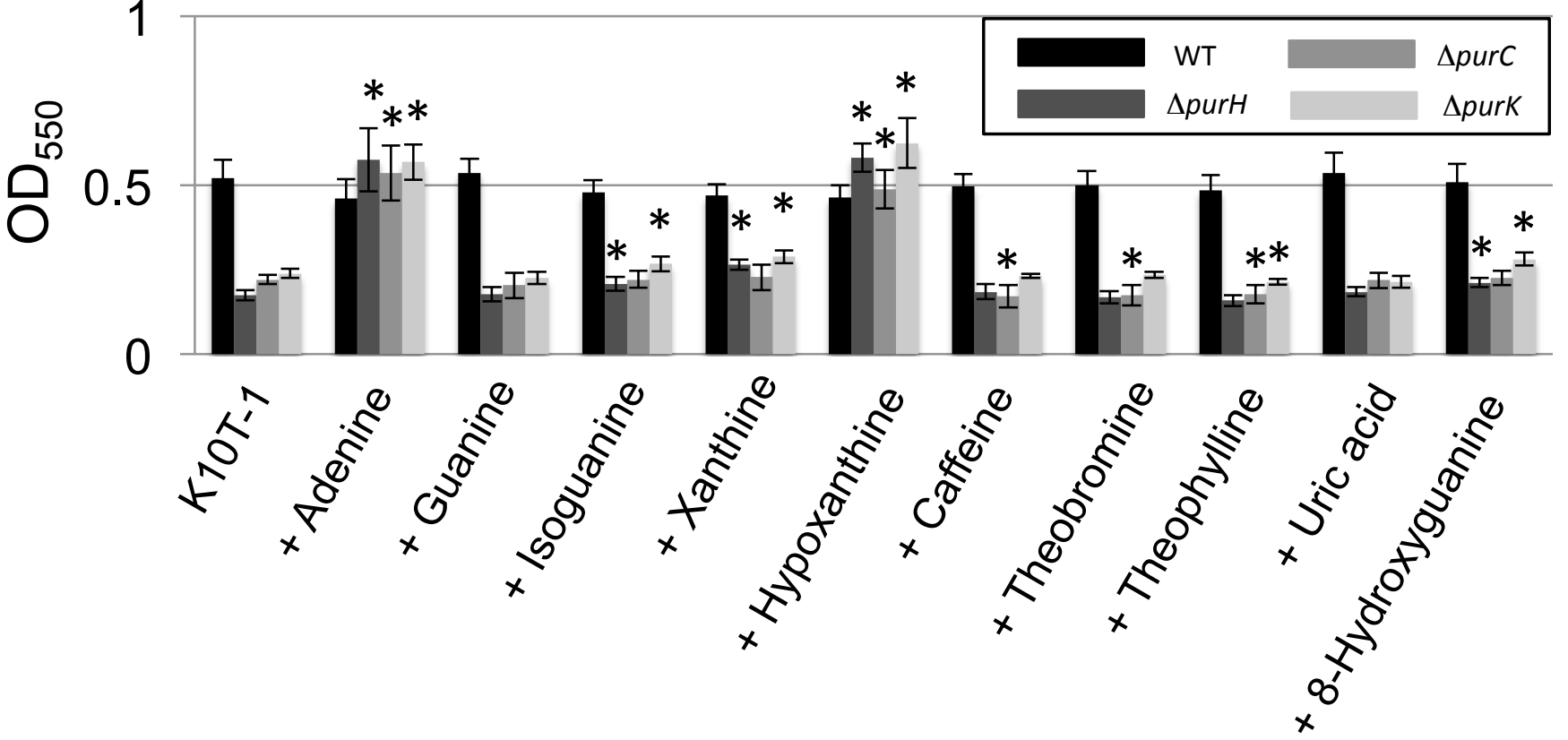

D

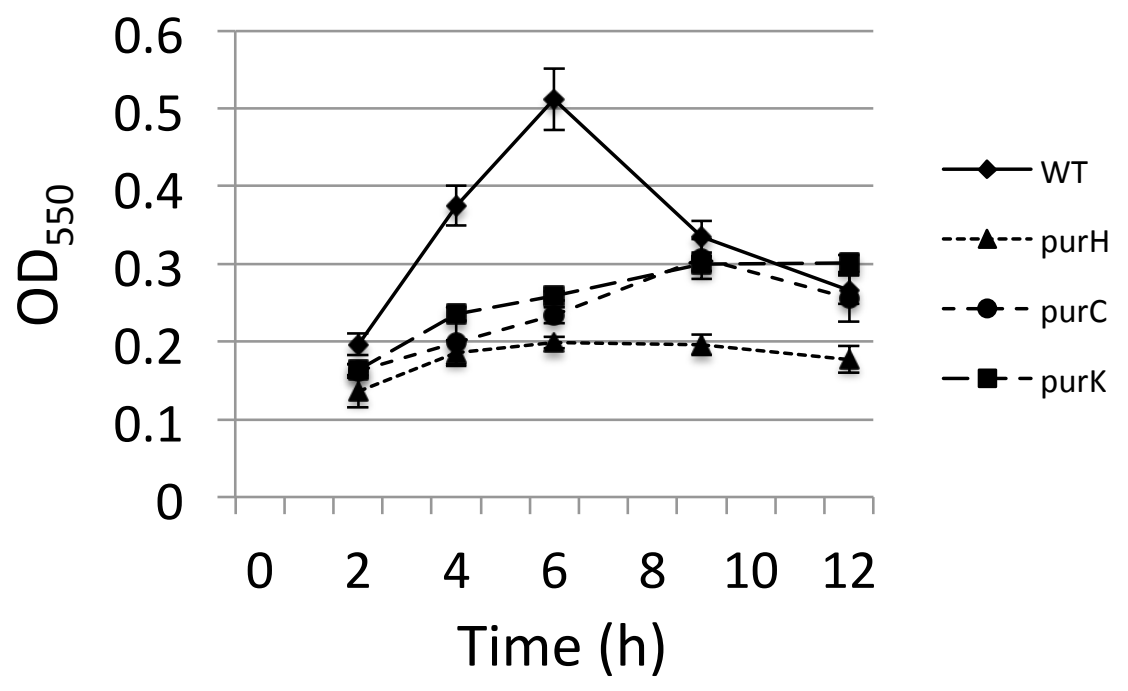




\section{Figure 2 (on next page)}

Analyses of the planktonic and biofilms cells and quantification of cell surface LapA.

(A) Time-dependent change in cell density for WT ( $\bullet$ ) and the mutants (DpurH ( $\mathbf{\Delta}), \Delta \operatorname{purC}(\bullet)$ and $\Delta$ purK ( $\square$ )) in K10T-1 medium (absorbance at 590nm; mean $\pm S D, n=8$ ). The experiments were performed in the same volume as the biofilm formation assay, and were done in at least twice. (B) Time-dependent change in cell density for WT ( $)$ and the mutants $(\Delta \operatorname{purH}(\mathbf{\Lambda}), \triangle \operatorname{purC}(\mathbf{\bullet})$ and $\Delta \operatorname{purK}(\mathbf{\square}))$ when $1 \mathrm{mM}$ adenine was added to the medium (absorbance at 590nm; mean $\pm S D, n=8$ ). The experiments were done duplicate. The asterisks $(*)$ show statistically significant difference in number of the cells compared to that of WT ( $P<0.01$ in two-tailed Student's t-test assuming equal variance). (C) Measurements of total amounts of ATP in planktonic and biofilm cells for WT and mutants. Data are the means $\mathrm{RLU} \pm \mathrm{SD}(n=4)$. The asterisks $(*)$ show statistically significant difference in RLU relative to that of WT ( $P<0.01$ in two-tailed Student's t-test assuming equal variance). (D) Effect of the mutations on numbers of the cells in inoculums, biofilms and supernatants. The black, dark gray, and light gray bars indicate number of the cells in the inoculum ( $t=0 \mathrm{hrs}$ ), biofilms ( $\mathrm{t}=6 \mathrm{hrs}$ ), and supernatants ( $\mathrm{t}=6 \mathrm{hrs}$ ), respectively, for WT and the three mutants ( $\triangle \mathrm{purH}$, $\triangle$ purC and $\triangle p u r C)$; mean $\pm S D, n=4$. The experiments were done duplicate. Significant differences $(P<0.01)$ were determined by two-tailed Student's t-test assuming equal variance and indicated with asterisks ${ }^{*}$ ). (E) Cell surface LapA quantification at $1 \mathrm{hr}$. Strains were grown statically in K10T-1 with or without $0.2 \mathrm{mM}$ adenine for 1 hour, then harvested and probed for LapA-HA by dot blotting. All mutants showed a significant reduction in LapA level compared to WT ( $* \mathrm{P}<0.001$, Wilcoxon Sum Rank Test). Addition of adenine significantly increased LapA on all of the mutants, and in the case of purH restored a WT level of LapA. (F) Cell surface LapA quantification at $9 \mathrm{hrs}$. Strains were grown for 9 hours with aeration in K10T-1, then LapA measured as in (E). All mutants showed a significant reduction in LapA level compared to WT (*P<0.0001, Wilcoxon Sum Rank Test). 
Figure 2

A

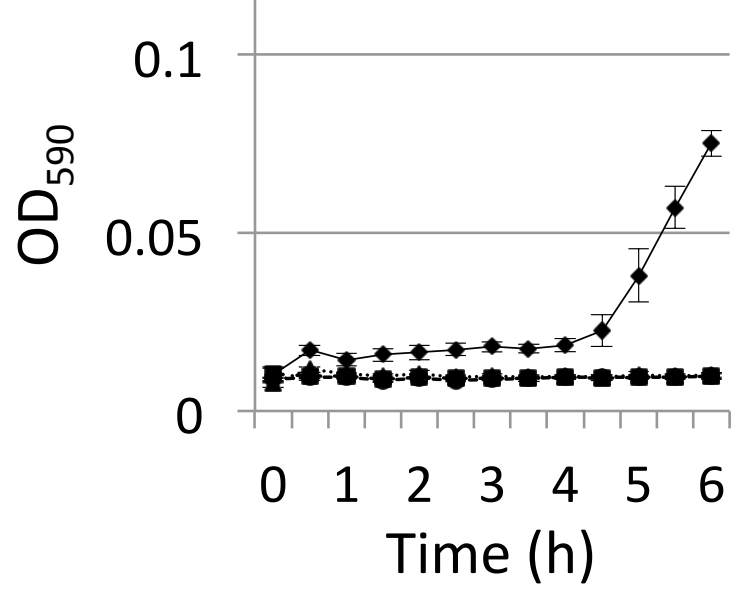

C

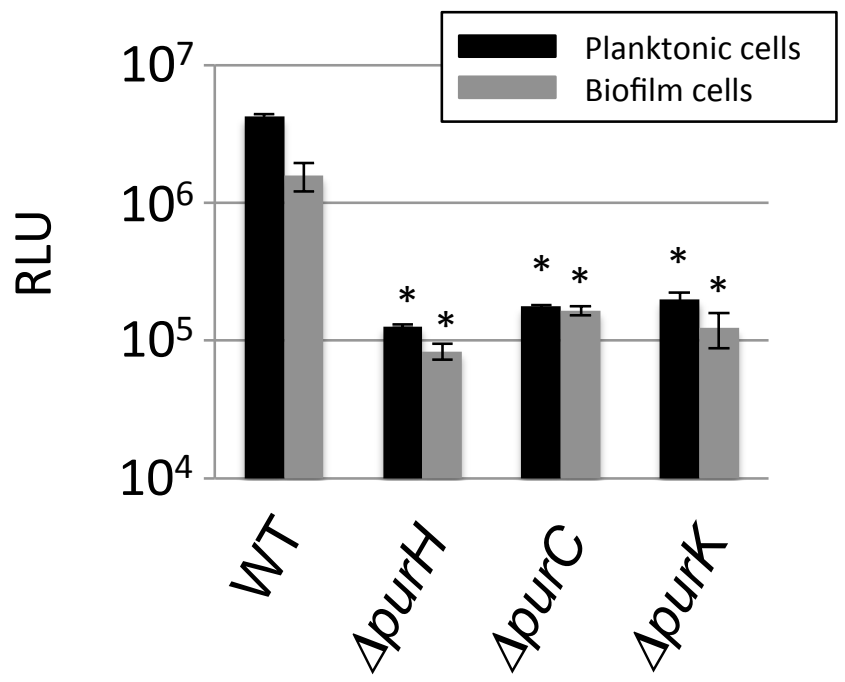

$E$

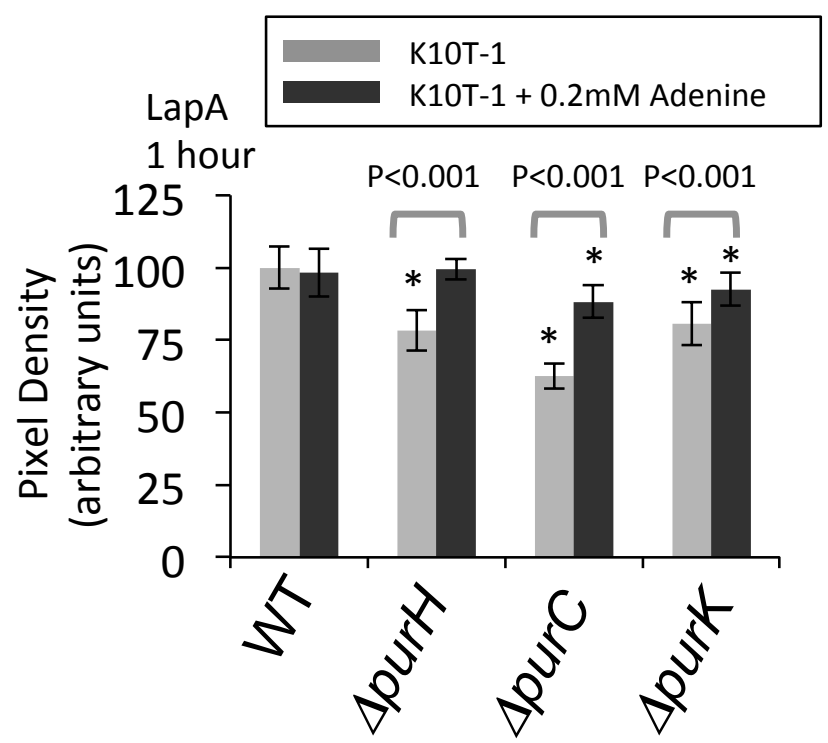

B

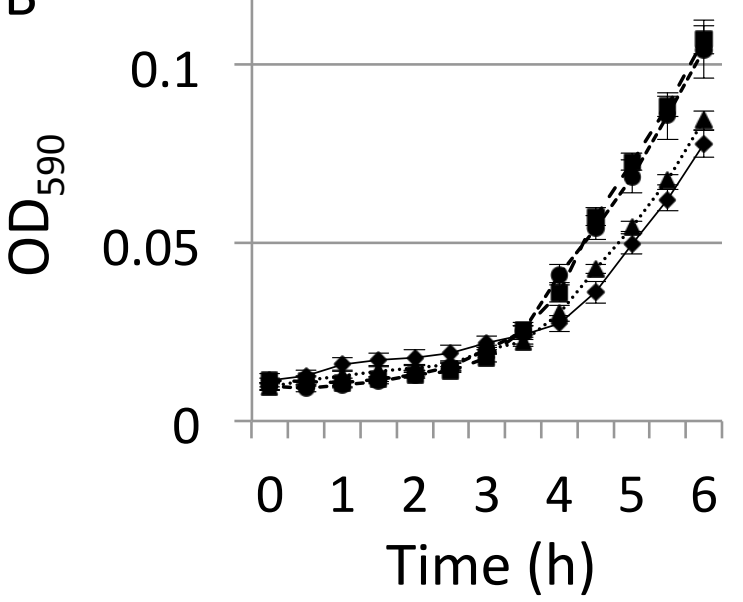

D

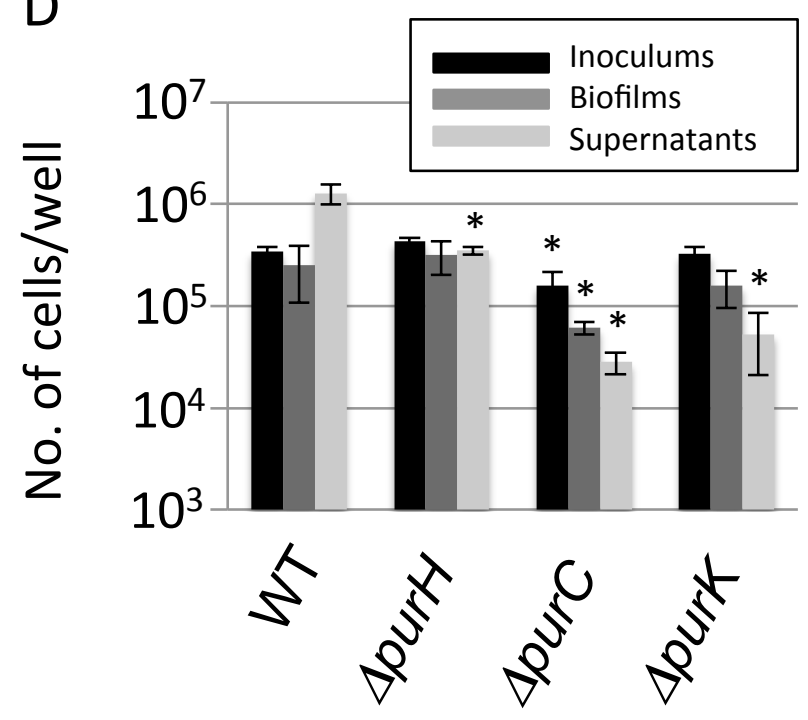

$\mathrm{F}$

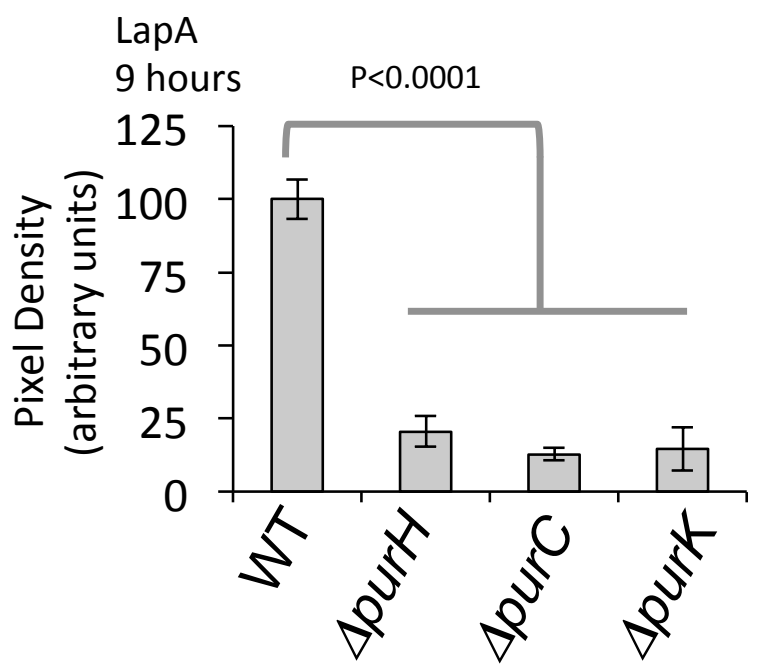




\section{Figure 3 (on next page)}

Effect of the mutations on sizes of the biofilm cells.

(A) Scanning electron micrographs showing the differences in cell size of the biofilm cells between WT and the mutants ( $\triangle p u r H, \Delta p u r C$ and $\triangle p u r C$ ) in K10T-1 at 6hrs. The white bars represent $5 \mu \mathrm{m}$. The histograms shown below the micrographs are the output data from the Phenom ParticleMetric software shown with minor modifications on the axes and labels for clarity. The medians of the circumscribed circle diameters are indicated on the each histogram. The analyses were performed three times for each strain, and one of them is shown in the figure. Five to fifteen SEM images were used to collect the circumscribed circle diameters of more than 1,000 cells for each analysis. (B) Scanning electron micrographs showing changes in the cell sizes upon supplementation of $0.2 \mathrm{mM}$ adenine into K10T-1 at $6 \mathrm{hrs}$. The white bars indicate $5 \mu \mathrm{m}$. The histograms labeled with the medians are shown below the micrographs. The analyses were performed three times for each strain, and one of them is represented. To collect the circumscribed circle diameters of over 1,000 cells, five to seven SEM images were used. (C) Scanning electron micrographs showing changes in the cell sizes of the biofilm cells when cultured at 9hrs in K10T-1. The white bars indicate $5 \mu \mathrm{m}$. The histograms labeled with the medians are shown below the micrographs. The analyses were performed three times for each strain, and one of them is shown in the figure. Twelve to eighteen SEM images were used for the analyses. 
PeerJ

Manuscript to be reviewed

Figure 3

A 1. WT

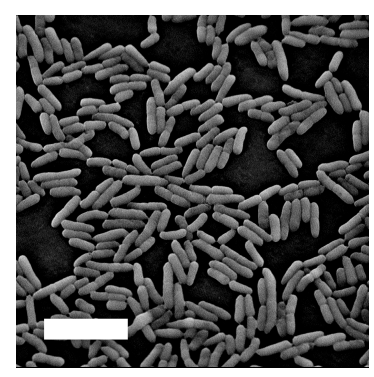

2. $\Delta$ purH

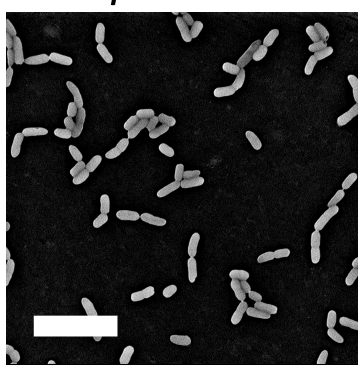

3. $\Delta p u r C$

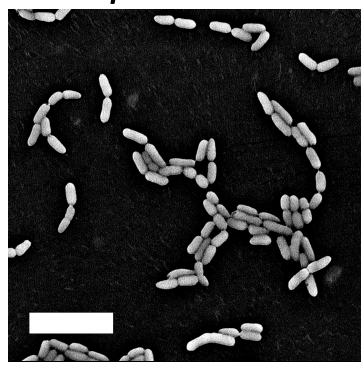

4. $\Delta p u r K$

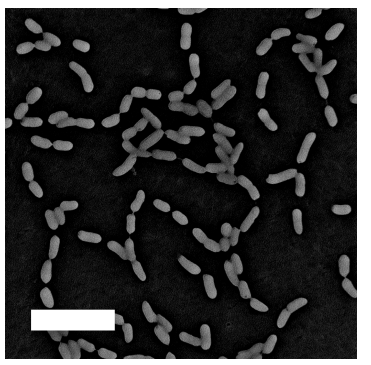

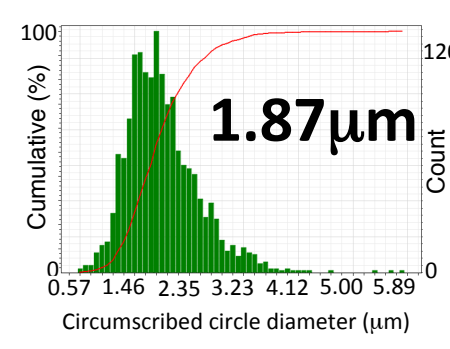
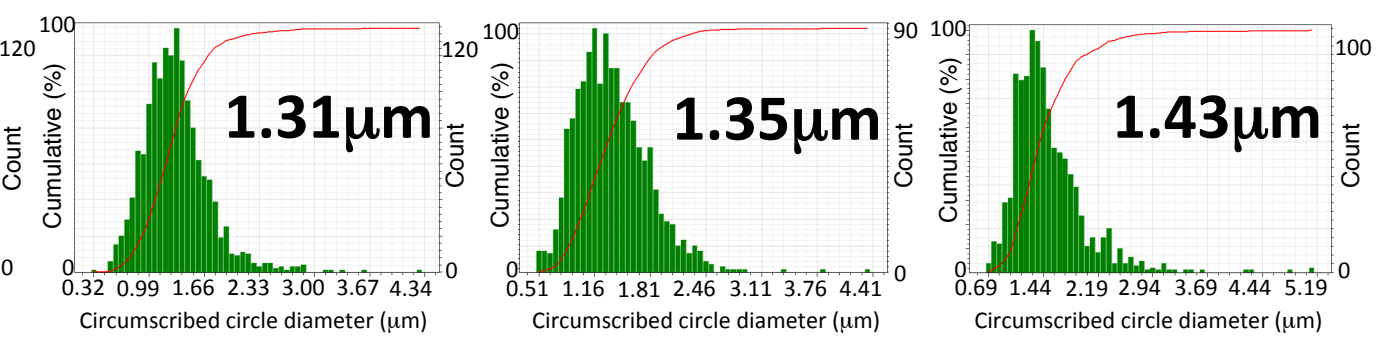

B 1. WT

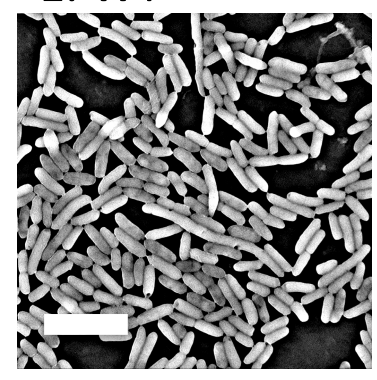

2. $\Delta$ purH

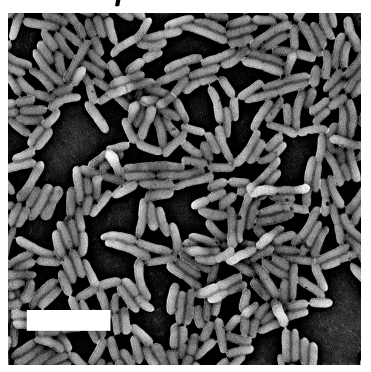

3. $\triangle p u r C$

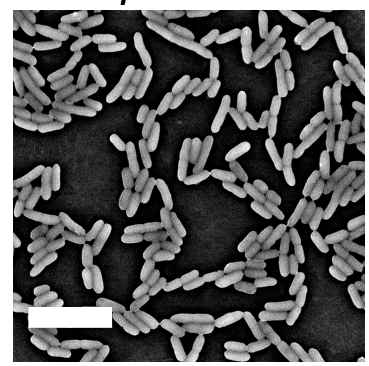

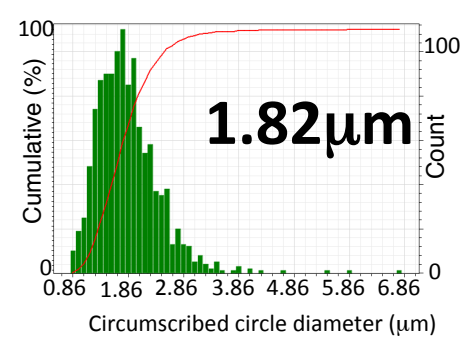

C 1. WT

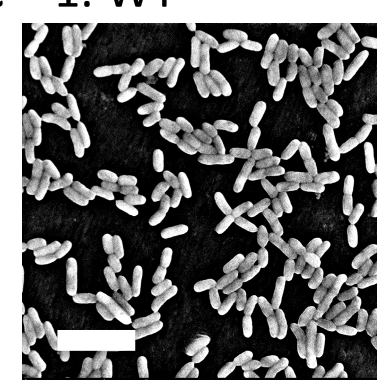

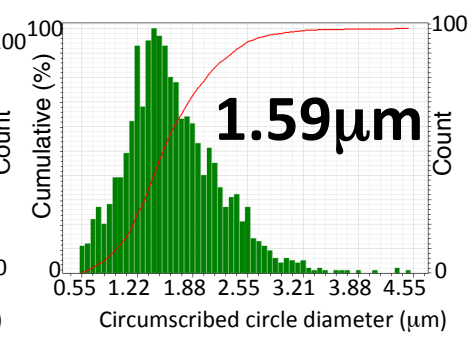

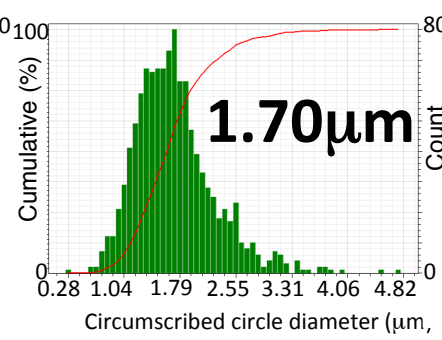

4. $\Delta$ purK
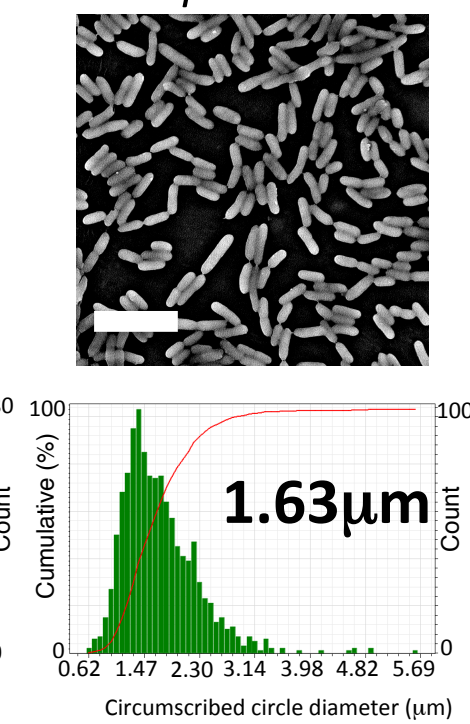

3. $\triangle p u r C$

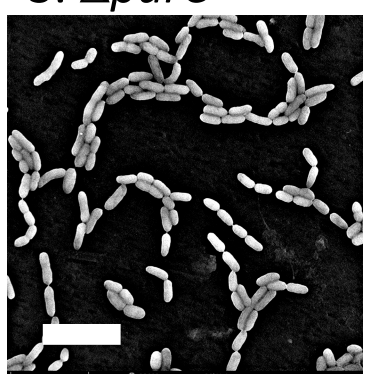

4. $\Delta$ purk
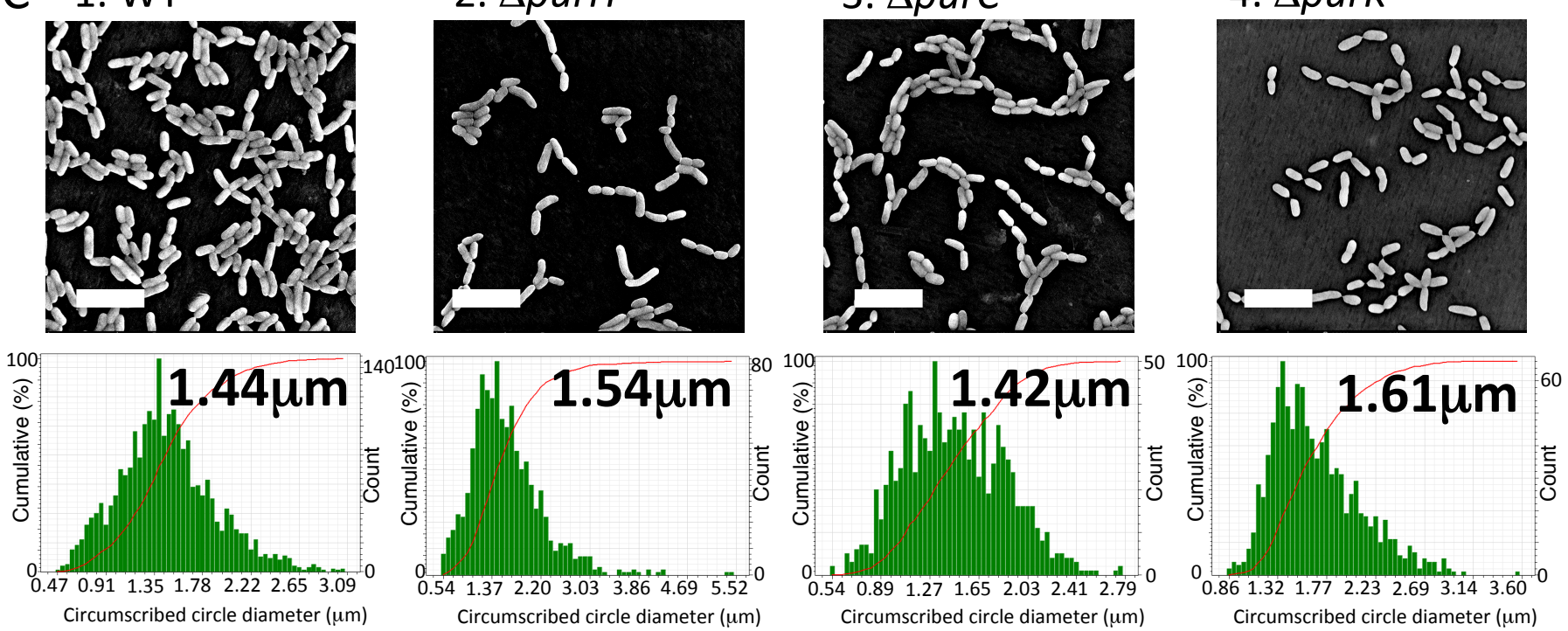


\section{Figure 4 (on next page)}

The size distributions for the planktonic and biofilm cells of WT and mutants obtained using a laser diffraction particle analyzer.

Panel A, B, C, and D show the size distribution for WT, $\Delta p u r H, \Delta p u r C$, and $\Delta p u r K$, respectively. For each panel, the size distributions for planktonic and biofilm cells cultured in K10T-1 medium are shown in solid and dotted lines, respectively. The dashed lines indicate the size distributions for the planktonic cells cultured in the medium with $0.2 \mathrm{mM}$ adenine. The measurements were performed three times for each, and one of the results are shown in the figure. See Table 2 for the statistics of the repeated measurements. 
Figure 4

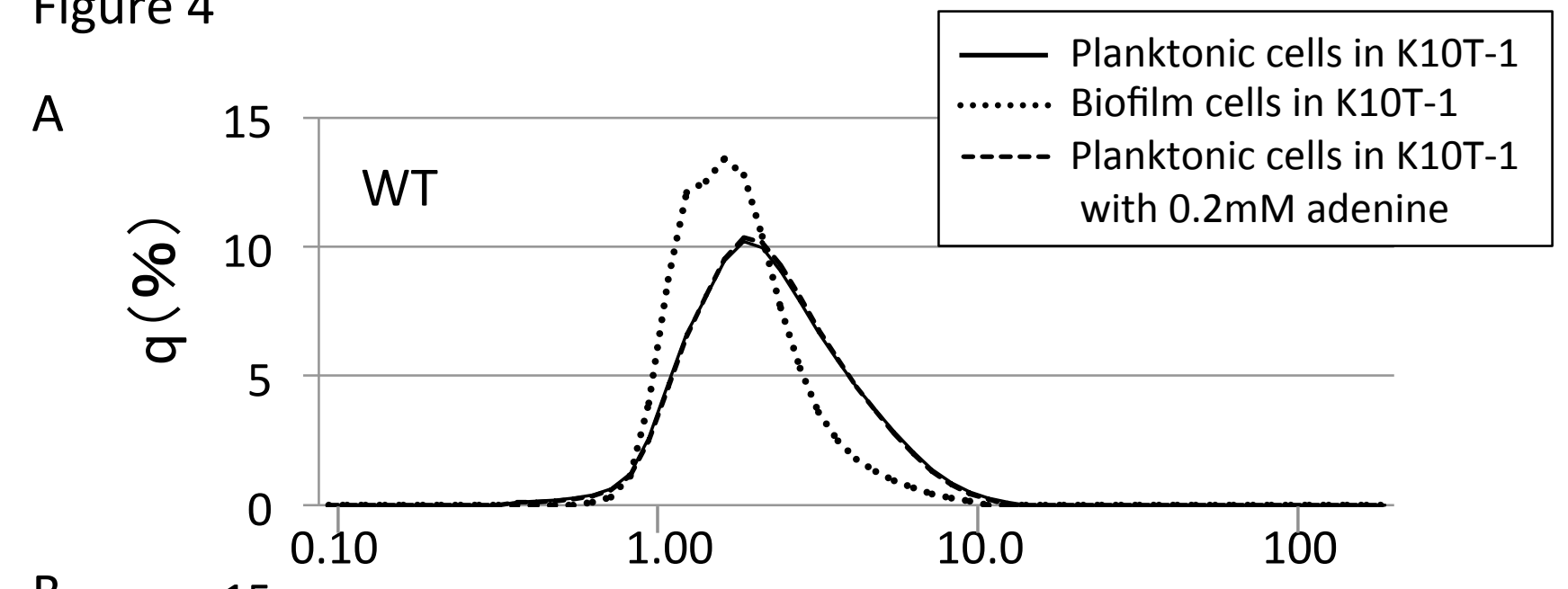

B

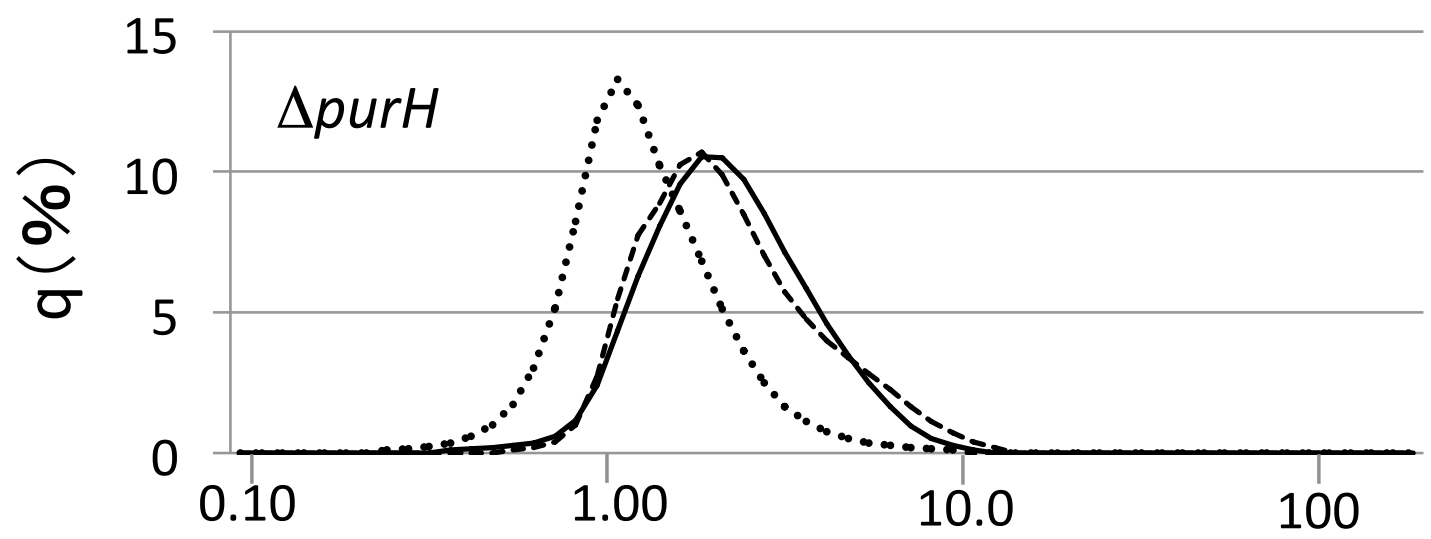

C

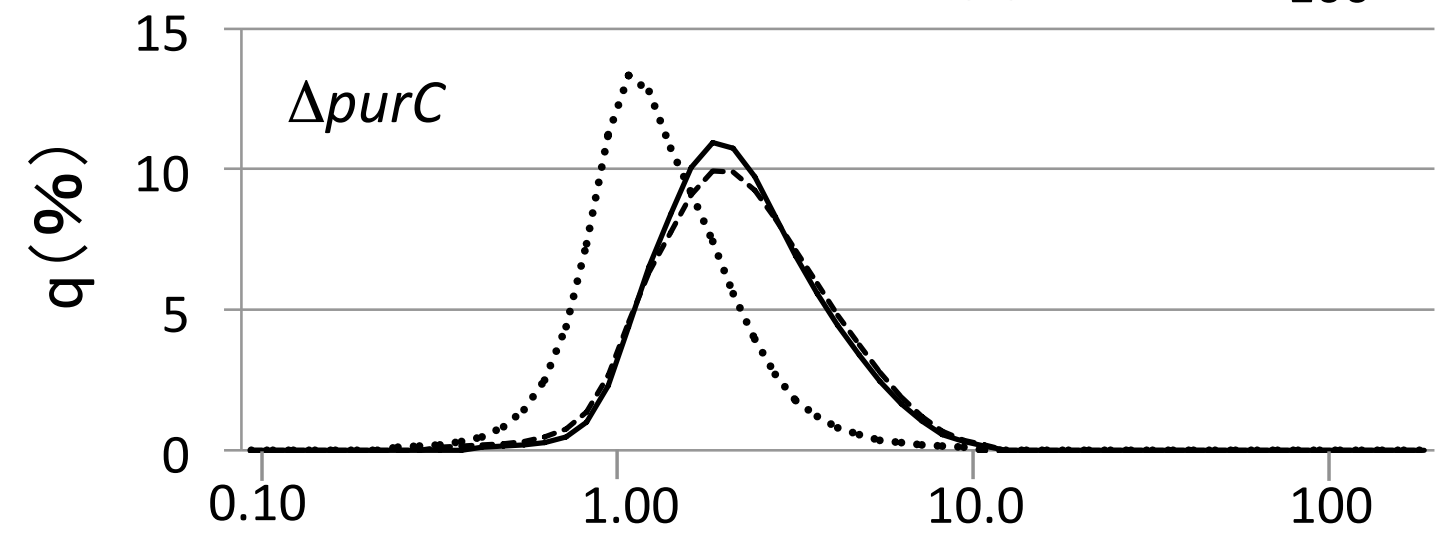

D

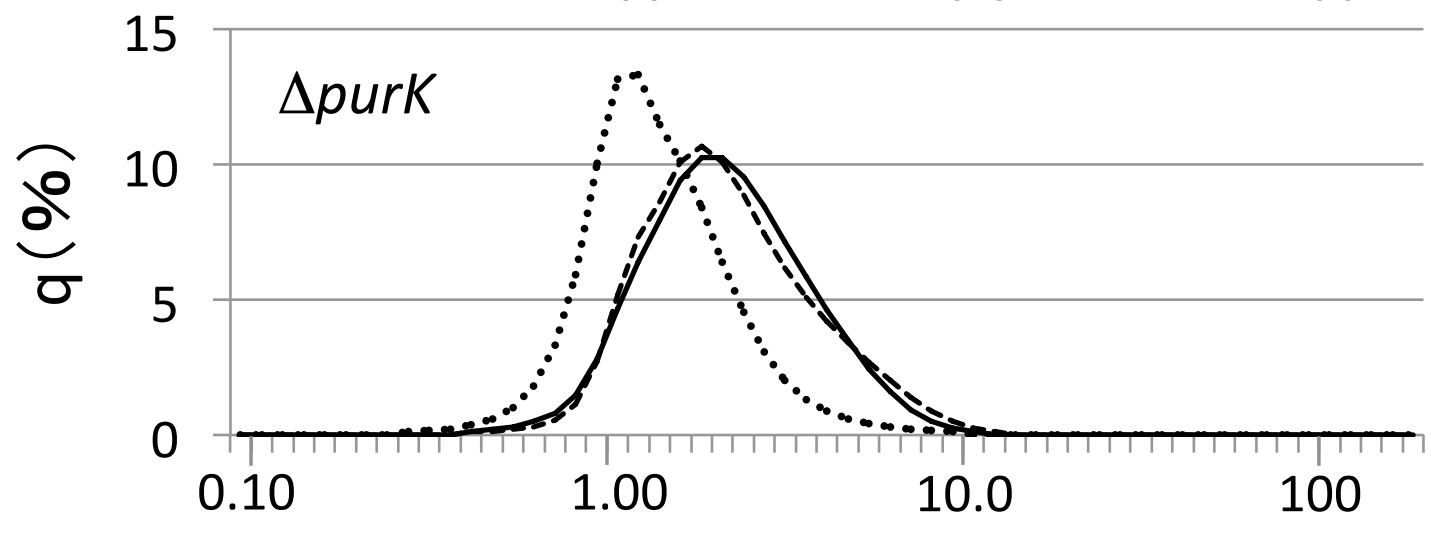

Diameter $(\mu \mathrm{m})$ 


\section{Table $\mathbf{1}$ (on next page)}

Summary of the circumscribed circle diameters $(\mu \mathrm{m})$ for WT and the mutants obtained by the SEM analyses.

The parentheses indicate the $P$ values obtained by the Wilcoxon Sum Rank test. ${ }^{a} P$ value when compared to the WT values in the same media. ${ }^{b} P$ value when compared to the same strain in K10T-1 medium at 6hrs. ${ }^{*}$ The asterisks indicate no statistically significant difference in size relative to that of WT in the same medium $(P>0.05)$. 
1 Table. 1. Summary of the circumscribed circle diameters $(\mu \mathrm{m})$ for $W T$ and the mutants 2 obtained by the SEM analyses.

\begin{tabular}{|c|c|c|c|c|c|}
\hline & & WT & $\Delta p u r H$ & $\Delta p u r C$ & $\Delta p u r K$ \\
\hline $\begin{array}{l}\text { K10T-1 } \\
\text { at 6hrs }\end{array}$ & $\begin{array}{c}\text { Median } \\
P(\text { Wilcoxon }) \\
\text { Mean } \pm \text { S.D. }\end{array}$ & $\begin{array}{c}1.87 \\
1.96 \pm 0.59\end{array}$ & $\begin{array}{c}1.31 \\
(<2.2 \mathrm{E}-16)^{\mathrm{a}} \\
1.35 \pm 0.39\end{array}$ & $\begin{array}{c}1.35 \\
(<2.2 \mathrm{E}-16)^{\mathrm{a}} \\
1.40 \pm 0.42\end{array}$ & $\begin{array}{c}1.43 \\
(<2.2 \mathrm{E}-16)^{\mathrm{a}} \\
1.53 \pm 0.48\end{array}$ \\
\hline $\begin{array}{c}+0.2 \mathrm{mM} \\
\text { adenine } \\
\text { at } 6 \mathrm{hrs}\end{array}$ & $\begin{array}{c}\text { Median } \\
P(\text { Wilcoxon }) \\
P(\text { Wilcoxon }) \\
\text { Mean } \pm \text { S.D. }\end{array}$ & $\begin{array}{c}1.82 \\
(0.002373)^{\mathrm{b}} \\
1.89 \pm 0.57\end{array}$ & $\begin{array}{c}1.59 \\
(<2.2 \mathrm{E}-16)^{\mathrm{a}} \\
(<2.2 \mathrm{E}-16)^{\mathrm{b}} \\
1.68 \pm 0.56\end{array}$ & $\begin{array}{c}1.70 \\
(2.99 \mathrm{E}-10)^{\mathrm{a}} \\
(<2.2 \mathrm{E}-16)^{\mathrm{b}} \\
1.76 \pm 0.54\end{array}$ & $\begin{array}{c}1.63 \\
(<2.2 \mathrm{E}-16)^{\mathrm{a}} \\
(<2.2 \mathrm{E}-16)^{\mathrm{b}} \\
1.74 \pm 0.56\end{array}$ \\
\hline $\begin{array}{l}\mathrm{K} 10 \mathrm{~T}-1 \\
\text { at } 9 \mathrm{hrs}\end{array}$ & $\begin{array}{c}\text { Median } \\
P(\text { Wilcoxon }) \\
P(\text { Wilcoxon }) \\
\text { Mean } \pm \text { S.D. }\end{array}$ & $\begin{array}{c}1.44 \\
(<2.2 \mathrm{E}-16)^{\mathrm{b}} \\
1.48 \pm 0.43\end{array}$ & $\begin{array}{c}1.54 \\
(2.36 \mathrm{E}-11)^{\mathrm{a}} \\
(<2.2 \mathrm{E}-16)^{\mathrm{b}} \\
1.65 \pm 0.60\end{array}$ & $\begin{array}{c}1.42 \\
(0.1079)^{\mathrm{a},{ }^{*}} \\
(9.98 \mathrm{E}-05)^{\mathrm{b}} \\
1.45 \pm 0.38\end{array}$ & $\begin{array}{c}1.61 \\
(2.20 \mathrm{E}-16)^{\mathrm{a}} \\
(<2.2 \mathrm{E}-16)^{\mathrm{b}} \\
1.69 \pm 0.40\end{array}$ \\
\hline
\end{tabular}

3 The parentheses indicate the $P$ values obtained by the Wilcoxon Sum Rank test.

$4 \quad{ }^{\text {a }} P$ value when compared to the WT values in the same media.

$5 \quad \mathrm{~b} P$ value when compared to the same strain in K10T-1 medium at 6hrs.

$6{ }^{*}$ The asterisks indicate no statistically significant difference in size relative to that of WT in the 7 same medium $(P>0.05)$. 


\section{Table 2 (on next page)}

Summary statistics for cell size of WT and mutants obtained by a laser diffraction particle analyzer.

Values represent mean \pm standard deviation for three independent measurements. ${ }^{a}$ The D50 is the median that is defined as the diameter where half of the population lies below this value. In the same way, 10 percent of the distribution lies below the D10, and 90 percent of the population lies below the D90. Standard deviations for D10 and D90 values were all below 0.05 . * Significantly smaller than planktonic cells of the same strain grown in K10T-1 by Student's t-test, $\mathrm{P}<0.002$. "Significantly smaller than WT biofilm cells by Student's t-test, $\mathrm{P}<0.001$. 
1 Table 2. Summary statistics for cell size of WT and mutants obtained by a laser diffraction

2 particle analyzer. Values represent mean \pm standard deviation for three independent

3 measurements.

\begin{tabular}{|c|c|c|c|c|c|}
\hline Conditions & Summary Statistics & WT & $\Delta p u r H$ & $\Delta p u r C$ & $\Delta p u r K$ \\
\hline Planktonic in & Mode size $(\mu \mathrm{m})$ & $1.86 \pm 5 \times 10^{-4}$ & $1.94 \pm 0.14$ & $1.94 \pm 0.14$ & $1.94 \pm 0.15$ \\
\hline \multirow[t]{4}{*}{$\mathrm{K} 10 \mathrm{~T}-1$ at $6 \mathrm{hrs}$} & $\mathrm{D} 10(\mu \mathrm{m})^{\mathrm{a}}$ & $1.14 \pm 4 \times 10^{-3}$ & $1.16 \pm 2 \times 10^{-3}$ & $1.18 \pm 6 \times 10^{-3}$ & $1.12 \pm 9 \times 10^{-3}$ \\
\hline & $\mathrm{D} 50(\mu \mathrm{m})^{\mathrm{a}}$ & $2.13 \pm 7 \times 10^{-3}$ & $2.14 \pm 2 \times 10^{-3}$ & $2.12 \pm 2 \times 10^{-2}$ & $2.12 \pm 6 \times 10^{-3}$ \\
\hline & $\mathrm{D} 90(\mu \mathrm{m})^{\mathrm{a}}$ & $4.78 \pm 2 \times 10^{-2}$ & $4.40 \pm 4 \times 10^{-3}$ & $4.41 \pm 5 \times 10^{-3}$ & $4.38 \pm 4 \times 10^{-3}$ \\
\hline & Mean size $(\mu \mathrm{m})$ & $2.62 \pm 9 \times 10^{-3}$ & $2.52 \pm 1 \times 10^{-3}$ & $2.52 \pm 4 \times 10^{-3}$ & $2.49 \pm 9 \times 10^{-4}$ \\
\hline Planktonic in & Mode size $(\mu \mathrm{m})$ & $1.86 \pm 6 \times 10^{-4}$ & $1.85 \pm 6 \times 10^{-4}$ & $2.03 \pm 0.15$ & $1.85 \pm 2 \times 10^{-3}$ \\
\hline $\mathrm{K} 10 \mathrm{~T}-1+$ & $\mathrm{D} 10(\mu \mathrm{m})^{\mathrm{a}}$ & $1.16 \pm 4 \times 10^{-4}$ & $1.15 \pm 2 \times 10^{-2}$ & $1.12 \pm 5 \times 10^{-3}$ & $1.14 \pm 1 \times 10^{-2}$ \\
\hline $0.2 \mathrm{mM}$ adenine & $\mathrm{D} 50(\mu \mathrm{m})^{\mathrm{a}}$ & $2.14 \pm 5 \times 10^{-3}$ & $2.04 \pm 1 \times 10^{-2}$ & $2.15 \pm 1 \times 10^{-2}$ & $2.07 \pm 2 \times 10^{-2}$ \\
\hline \multirow[t]{2}{*}{ at $6 \mathrm{hrs}$} & $\mathrm{D} 90(\mu \mathrm{m})^{\mathrm{a}}$ & $4.67 \pm 2 \times 10^{-2}$ & $4.94 \pm 4 \times 10^{-2}$ & $4.60 \pm 2 \times 10^{-2}$ & $4.71 \pm 5 \times 10^{-2}$ \\
\hline & Mean size $(\mu \mathrm{m})$ & $2.59 \pm 9 \times 10^{-3}$ & $2.62 \pm 2 \times 10^{-2}$ & $2.57 \pm 1 \times 10^{-3}$ & $2.56 \pm 2 \times 10^{-2}$ \\
\hline Biofilm in & Mode size $(\mu \mathrm{m})$ & $* 1.62 \pm 6 \times 10^{-4}$ & ${ }^{* \#} 1.08 \pm 6 \times 10^{-4}$ & ${ }^{* \#} 1.08 \pm 6 \times 10^{-5}$ & ${ }^{* \#} 1.13 \pm 8 \times 10^{-2}$ \\
\hline \multirow[t]{4}{*}{ K10T-1 at $6 \mathrm{hrs}$} & $\mathrm{D} 10(\mu \mathrm{m})^{\mathrm{a}}$ & $1.08 \pm 3 \times 10^{-3}$ & $0.72 \pm 1 \times 10^{-3}$ & $0.76 \pm 5 \times 10^{-4}$ & $0.77 \pm 3 \times 10^{-2}$ \\
\hline & $\mathrm{D} 50(\mu \mathrm{m})^{\mathrm{a}}$ & $1.69 \pm 6 \times 10^{-3}$ & $1.20 \pm 1 \times 10^{-3}$ & $1.25 \pm 4 \times 10^{-4}$ & $1.32 \pm 3 \times 10^{-2}$ \\
\hline & $\mathrm{D} 90(\mu \mathrm{m})^{\mathrm{a}}$ & $3.15 \pm 2 \times 10^{-2}$ & $2.37 \pm 1 \times 10^{-3}$ & $2.43 \pm 3 \times 10^{-4}$ & $2.49 \pm 3 \times 10^{-2}$ \\
\hline & Mean size $(\mu \mathrm{m})$ & $1.98 \pm 9 \times 10^{-3}$ & $1.44 \pm 1 \times 10^{-3}$ & $1.49 \pm 3 \times 10^{-4}$ & $1.52 \pm 3 \times 10^{-2}$ \\
\hline
\end{tabular}

4 a The D50 is the median that is defined as the diameter where half of the population lies below

5 this value. In the same way, 10 percent of the distribution lies below the D10, and 90 percent of

6 the population lies below the D90. Standard deviations for D10 and D90 values were all below 70.05 .

8 * Significantly smaller than planktonic cells of the same strain grown in K10T-1 by Student's t9 test, $\mathrm{P}<0.002$.

10 \# Significantly smaller than WT biofilm cells by Student's t-test, $\mathrm{P}<0.001$. 